\title{
Functional Specialization of Interneuron Dendrites: Identification of Action Potential Initiation Zone in Axonless Olfactory Bulb Granule Cells
}

\author{
R. Todd Pressler and Ben W. Strowbridge \\ Department of Neurosciences, Case Western Reserve University, Cleveland, Ohio 44106
}

Principal cells in the olfactory bulb (OB), mitral and tufted cells, play key roles in processing and then relaying sensory information to downstream cortical regions. How OB local circuits facilitate odor-specific responses during odor discrimination is not known but involves GABAergic inhibition mediated by axonless granule cells (GCs), the most abundant interneuron in the OB. Most previous work on GCs has focused on defining properties of distal apical dendrites where these interneurons form reciprocal dendrodendritic connections with principal cells. Less is known about the function of the proximal dendritic compartments. In the present study, we identified the likely action potentials (AP) initiation zone by comparing electrophysiological properties of rat (either sex) GCs with apical dendrites severed at different locations. We find that truncated GCs with long apical dendrites had active properties that were indistinguishable from intact GCs, generating full-height APs and short-latency low-threshold $\mathrm{Ca}^{2+}$ spikes. We then confirmed the presumed site of AP and low-threshold $\mathrm{Ca}^{2+}$ spike initiation in the proximal apical dendrite using two-photon $\mathrm{Ca}^{2+}$ photometry and focal TTX application. These results suggest that GCs incorporate two separate pathways for processing synaptic inputs: an already established dendrodendritic input to the distal apical dendrite and a novel pathway in which the cell body integrates proximal synaptic inputs, leading to spike generation in the proximal apical dendrite. Spikes generated by the proximal pathway likely enables GCs to regulate lateral inhibition by defining time windows when lateral inhibition is functional.

Key words: two-photon imaging; brain slice; dendrites; interneuron; olfaction; patch-clamp recording

Significance Statement

The olfactory bulb plays a central role in processing sensory input transduced by receptor neurons. How local circuits in the bulb function to facilitate sensory processing during odor discrimination is not known but appears to involve inhibition mediated by granule cells, axonless GABAergic interneurons. Little is known about the active conductances in granule cells including where action potentials originate. Using a variety of experimental approaches, we find the $\mathrm{Na}^{+}$-based action potentials originate in the proximal apical dendrite, a region targeted by cortical feedback afferents. We also find evidence for high expression of low-voltage activated $\mathrm{Ca}^{2+}$ channels in the same region, intrinsic currents that enable GCs to spike rapidly in response to sensory input during each sniff cycle.

\section{Introduction}

Sensory information in the olfactory system transduced by receptor neurons is initially processed in the olfactory bulb (OB) through synaptic interactions between principal cells and GABAergic

\footnotetext{
Received July 23, 2019; revised Sept. 25, 2019; accepted Sept. 29, 2019.

Author contributions: R.T.P. and B.W.S. designed research; R.T.P. performed research; R.T.P. and B.W.S. analyzed data; R.T.P. and B.W.S. wrote the paper.

This work was supported by NIH Grants R01-DC04285 and R21-NS103182 to B.W.S. and R03-DC913641 to R.T.P.

We thank Dr. Dan Wesson for helpful discussions related to this project and Dr. Chris Ford for providing helpful comments on the paper.

The authors declare no competing financial interests.

Correspondence should be addressed to Ben W. Strowbridge at bens@case.edu.

https://doi.org/10.1523/JNEUROSCI.1763-19.2019

Copyright $\odot 2019$ the authors
}

interneurons. How local OB inhibitory circuits facilitate neural representations of sensory input patterns during odor discrimination (Abraham et al., 2010) is poorly understood in part because few previous studies focused on defining the functional properties of granule cells (GCs), the primary interneuron in the mammalian OB. Olfactory GCs lack axons (Shepherd et al., 2007) and, instead, release GABA from spines on their distal apical dendritic arbor at specialized dendrodendritic (DD) synapses (Rall et al., 1966; Isaacson and Strowbridge, 1998). Why neural computations performed by the $\mathrm{OB}$ rely on axonless interneurons and DD synaptic circuits is not well understood experimentally but has been explored using computational models (Cleland, 2014; Yu et al., 2014). 
Previous studies of GC physiology using intracellular recording methods described frequent spontaneous EPSPs (Cang and Isaacson, 2003; Youngstrom and Strowbridge, 2015) attributed to DD synaptic inputs from spontaneously active mitral and tufted cells. (Olfactory principal neurons often fire spontaneously even in in vitro brain slices; Castillo et al., 1999; Friedman and Strowbridge, 2003; Schmidt and Strowbridge, 2014.) In addition to DD synaptic input that selectively targets the distal apical dendrites of GCs, the only other established glutamatergic input to GCs arises from piriform cortex and related olfactory cortical structures (de Olmos et al., 1978; Shipley and Adamek, 1984; Gao and Strowbridge, 2009; Markopoulos et al., 2012) and terminates on the proximal apical dendrite (Balu et al., 2007). GCs also have spines along their basal dendrites (Price and Powell, 1970a; Schneider and Macrides, 1978) though the source and function of these synaptic inputs has not been established.

Relatively little is known about the basic electrophysiology of GCs, including where action potentials (APs) are initiated and whether APs are actively propagated throughout the dendritic arbor. Retinal amacrine cells may be a potential analog to GCs, which also lack axons and generate APs at anatomically distinct regions of their proximal dendrite (Cook and Werblin, 1994; Wu et al., 2011; Cembrowski et al., 2012). GCs also contain a wide variety of active conductances, including low-voltage activated (LVA) $\mathrm{Ca}^{2+}$ channels (Egger et al., 2003, 2005; Pinato and Midtgaard, 2003, 2005; Inoue and Strowbridge, 2008), though the spatial organization of this intrinsic current also has not been defined.

The goal of the present study was to determine how functional properties are organized spatially within GCs. The narrow width of apical and basal dendrites of GCs make it technically challenging to answer this question directly using dendritic or paired somatic/dendritic patch-clamp recordings (Stuart et al., 1997a,b; Magee and Johnston, 1995, in pyramidal cells). Instead, we used a two-part approach by first defining how electrophysiological properties vary with apical and basal dendritic extent. This comparative approach took advantage of intracellular recordings from GCs with apical dendrites that were severed before they entered the external plexiform layer (EPL), minimizing the large source of spontaneous depolarizations arising from DD synaptic inputs. Our comparisons of different subpopulations of truncated GCs suggest that the proximal apical dendrite is the likely location of both AP initiation and the LVA $\mathrm{Ca}^{2+}$ channels that generate low-threshold spikes in GCs. In the second part of this study, we confirmed these results using focal application of specific pharmacological agents and with two-photon $\mathrm{Ca}^{2+}$ photometry in intact GCs. We also find that excitatory inputs terminate frequently on GC basal dendrites. Together, our results suggest that GCs incorporates two separate pathways for processing excitatory input: the well known DD input to the distal apical dendrite and a novel pathway in which the GC somata integrates basalar synaptic inputs, leading to spike generation in the proximal apical dendrite. Proximally-triggered spikes likely regulate lateral inhibition in the $\mathrm{OB}$ by defining narrow time windows when NMDARs that govern when DD inhibition are functional.

\section{Materials and Methods}

Animals. Horizontal OB slices (300 $\mu \mathrm{m}$ thick) were prepared from postnatal day (P)14-P25 Sprague-Dawley rats of either sex anesthetized with ketamine, as described previously (Pressler and Strowbridge, 2006; Gao and Strowbridge, 2009). OB slices were incubated initially at $30^{\circ} \mathrm{C}$ for 30 min and then maintained at room temperature until use. All experiments were performed in accordance with the guidelines approved by the Case
Western Reserve University Animal Care and Use Committee. During recording sessions slices were placed in a submersion chamber and perfused with oxygenated artificial CSF (ACSF) at a rate of $1.5 \mathrm{ml} / \mathrm{min}$. Recordings were made between $29^{\circ}$ and $31^{\circ} \mathrm{C}$. ACSF consisted of (in mM): $124 \mathrm{NaCl}, 3 \mathrm{KCl}, 1.23 \mathrm{NaH}_{2} \mathrm{PO}_{4}, 1.2 \mathrm{MgSO}_{4}, 26 \mathrm{NaHCO}_{3}, 10$ dextrose, $2.5 \mathrm{CaCl}_{2}$, equilibrated with $95 \% \mathrm{O}_{2} / 5 \% \mathrm{CO}_{2}$. All drugs except tetrodotoxin (TTX) were added to the submerged recording chamber by changing the external solution source.

All neurons recorded were visualized using live two-photon imaging to facilitate categorization as either "intact GCs", with apical dendrites that passed through the mitral cell layer (MCL) and then ramified within the EPL $(N=33)$ or "truncated GCs", with apical dendrites that were severed within the GC layer (GCL; $N=67)$ or MCL $(N=4)$. Both intact and truncated GCs were recorded in the same set of experiments. Truncated GCs were generated either as a result of slice preparation $(N=56)$ or following a visualized micropuncture of the GCL region using the tip of a 25 gauge needle mounted on a motorized manipulator $(N=15)$. We combined both types of recordings in the truncated group. We also recorded from another set of visualized intact GCs $(N=69)$ to perform the mechanistic experiments described in this study (e.g., $\mathrm{Ca}^{2+}$ photometry and pharmacological tests).

Electrophysiology. Recordings were performed in a submerged chamber maintained at $30^{\circ} \mathrm{C}$ and perfused with an extracellular solution containing the following (in $\mathrm{mm}$ ): $124 \mathrm{NaCl}, 3 \mathrm{KCl}, 1.23 \mathrm{NaH}_{2} \mathrm{PO}_{4}, 1.2$ $\mathrm{MgSO}_{4}, 26 \mathrm{NaHCO}_{3}, 10$ dextrose, and $2.5 \mathrm{CaCl}_{2}$, equilibrated with $95 \%$ $\mathrm{O}_{2} / 5 \% \mathrm{CO}_{2}$. Whole-cell patch-clamp recordings were made using AxoPatch 1D amplifiers (Molecular Devices) and borosilicate glass pipettes (3-7 M $\Omega$ ) under infrared differential contrast microscopy (IR-DIC) video microscopy. Under current-clamp conditions, recording electrodes contained the following (in $\mathrm{mm}$ ): 140 Kmethylsulfate, $4 \mathrm{NaCl}, 10$ HEPES, 0.2 EGTA, 4 MgATP, $0.3 \mathrm{Na}_{3} \mathrm{GTP}, 10$ phosphocreatine di-tris. AlexaFluor 594 (100-150 $\mu \mathrm{M}$; Invitrogen) was also included routinely to permit visualization of the recorded neuron using live two-photon imaging, as described previously (Pressler and Strowbridge, 2006, 2017). Internal solutions were adjusted to $\mathrm{pH} 7.3$ and $290 \mathrm{mOsm}$.

Intracellular recordings were low-pass filtered at $5 \mathrm{kHz}$ (FLA-01, Cygus Technology) and acquired at $10 \mathrm{kHz}$ using an ITC-18 interface (Instrutech/HEKA) and custom Visual Basic.NET and Python software on a Windows 7-based personal computer. Intracellular recordings were not corrected for the liquid junction potential. Unless noted, all drugs were obtained from Tocris Bioscience except for TTX, which was purchased from Calbiochem.

For TTX focal application (see Fig. 5), a second patch pipette filled with saline solution containing AlexaFluor $594(100 \mu \mathrm{M})$ dissolved in 124 $\mathrm{mm} \mathrm{NaCl}, 3 \mathrm{~mm} \mathrm{KCl}$, and $10 \mathrm{~mm}$ HEPES, pH 7.4, and was used to focally apply brief puffs of $5 \mu \mathrm{M}$ TTX onto spatially restricted portions of the apical or basal dendritic arbor, or the soma. Focal application of TTX was guided using live two-photon imaging with the puffing pipette tip positioned within 5-10 $\mu \mathrm{m}$ of the GC process.

Data analysis and statistics. We selected for GCs by recording from neurons with small, elongated ("egg shaped") cell bodies in the GCL imaged using IR-DIC video microscopy and a $60 \times$ water-immersion objective. Although several other interneuron classes have been reported in this layer (Pressler and Strowbridge, 2006; Eyre et al., 2009; Pressler et al., 2013), these other cell types all have larger cell bodies than GCs and are typical found outside the stereotyped stripes of GC somata. We also confirmed GC-like morphology in all recordings using live two-photon imaging.

Input resistance was assayed by measuring the maximal hyperpolarization evoked by $1 \mathrm{~s}$ duration current steps applied from a holding potential of $-70 \mathrm{mV}$. AP threshold was defined by the voltage at $10 \%$ of the maximal membrane potential slope. AP width was a calculated from the time required for the repolarization phase to pass the threshold voltage. The small differences in the number of GCs reported in different experiments within this study reflected occasional recordings in which it was impossible to determine the extent of the basal dendrite because of inadequate $z$-stack coverage or when technical problems prevented analysis of responses to current steps necessary to assay input resistance or AP properties. In most analyses, we examined the length of the primary 
apical dendrite as very few truncated GCs generated collaterals of apical dendrite within the GCL. Analyses of the basal arbor were based on the total basal dendrite extent estimated by combining the lengths of each primary basal dendrite.

Spontaneous EPSPs were detected using custom Python programs that implemented an "up-jerk" detection algorithm described previously (Larimer and Strowbridge, 2008; Hyde and Strowbridge, 2012). All detected events were confirmed by visual inspection. All data analysis was performed using Python 3 scripts. Unless otherwise noted, data are presented as mean \pm SEM. $P$ values reported from repeated tests of the different divisions from the same dataset were adjusted to reflect the Bonferroni correction for multiple comparisons.

Nonraster two-photon $\mathrm{Ca}^{2+}$ photometry. We used a nonraster two-photon scanning method to assay $\mathrm{Ca}^{2+}$ indicator fluorescence in small regions of the GC dendritic arbor. In these experiments, we used an internal solution without EGTA that contained the following (in mM): 140 KMethylsulfate, $4 \mathrm{NaCl}, 10$ HEPES, 4 MgATP, $0.3 \mathrm{Na}_{3}$ GTP, 10 phosphocreatine ditris. In addition, we added an inert fluorescent dye, AlexaFluor $594(7.5 \mu \mathrm{M})$, to visualize GC morphology and an organic $\mathrm{Ca}^{2+}$ indicator dye (Cal-520, $100 \mu \mathrm{M}$; AAT Bioquest). Because only a single photomultiplier tube (PMT) detector with a broad range emission filter (BG39 Schott glass) was used in this study, both AlexaFluor 594 and Cal520 signals were combined in the PMT (H7422P-40, Hamamatsu) output.

We initially obtained a conventional image of the relevant part of the neuron using standard two-photon raster scanning methods and a $60 \times$ objective (Pressler and Strowbridge, 2006, 2017; Balu et al., 2007). We then computed the $\mathrm{X}$ and $\mathrm{Y}$ galvanometer command voltages that corresponded to the center of the area of interest on a GC dendrite or cell body. Custom software (https://github.com/Strow bridgeLab/Toronado-Laser-Scanning) then generated new galvanometer $\mathrm{X}$ and $\mathrm{Y}$ scan patterns that forced the excitation focal spot to repetitively circle around this center point at 5000 revolutions/s. Circle diameters were optimized in pilot recordings and then were fixed at $70 \mathrm{~nm}$ for the experiments in this study. For both raster and nonraster scan modes, we included 1:4 passive resistor dividers on the $\mathrm{X}$ and $\mathrm{Y}$ inputs to the galvanometer controller modules to facilitate small amplitude $(\sim 5 \mathrm{mV})$ scan commands. We recorded the position sensor output of the galvanometer controller in pilot experiments to verify that the intended scan pattern was generated.

The same PMT current preamplifier (SR-570, Stanford Research Systems) was used in both raster and nonraster modes but with different settings optimized for either high-bandwidth signals (for raster scanning) or high-sensitivity, low-noise signals (for nonraster photometry scanning). We attenuated scan-related noise by filtering the output of the current preamplifier at a frequency lower than the circling frequency ( 1 $\mathrm{kHz}, 8$ pole Bessel low-pass, FLA-01, Cygnus Technology) and by introducing random phase delays at the start of the scan patterns on each trial and then averaging three to five AP-aligned episodes. During nonraster scans, the PMT signal was acquired on the same data acquisition device (ITC-18) used to record the electrophysiological signals.
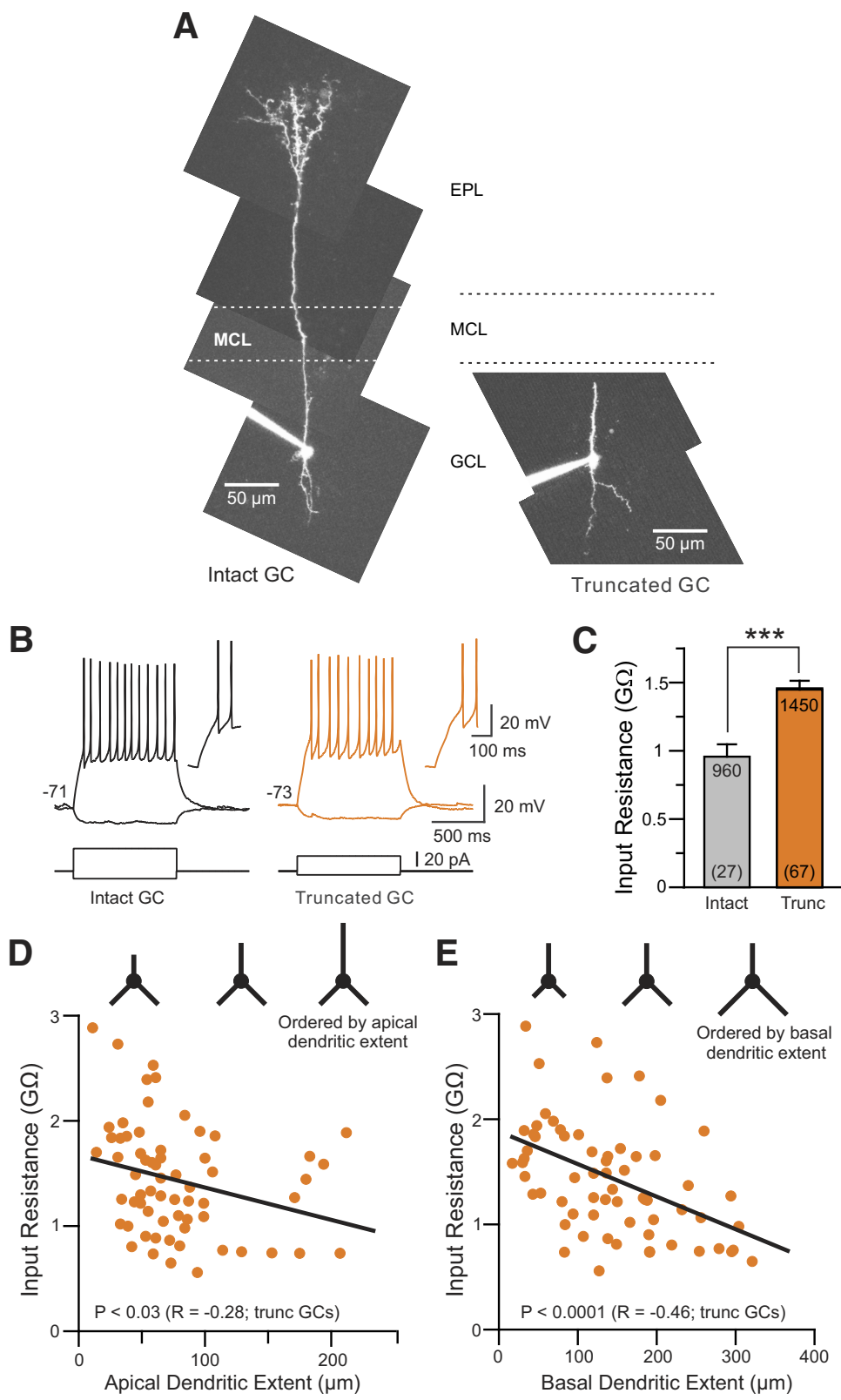

Figure 1. Intrinsic properties of intact and truncated GCS. A, Two-Photon z-stack montages illustrating an intact GC (left) and a truncated GC with no apical dendrite arbor in the EPL (right). $\boldsymbol{B}$, Example responses to depolarizing and hyperpolarizing current steps from intact (black traces) and truncated GCs (orange). Same two GCs shown in $\boldsymbol{A}$. Inset, Initial two APs at a faster sweep group indicated near bottom of bars and group means indicated near top of bars. $\boldsymbol{D}$, Plot of input resistance versus apical dendrite extent in truncated GCs. Linear regression superimposed with fit parameters indicated in illustration. $\boldsymbol{E}$, Plot of input resistance versus extent of basal dendritic arbor in the same group of truncated GCs shown in $\boldsymbol{D}$.

\section{Results}

The primary focus of this study is to understand the functional properties of the proximal dendrites of OB GCs. In intact GCs, frequent spontaneous EPSPs originating from distal DD inputs dominate the physiological properties of these interneurons. By taking advantage of "truncated GCs" (Fig. 1A) with short apical dendrites, we hypothesize that we can define the electrophysiological properties of proximal processes without the confounds of frequent synaptic inputs and intrinsic currents located in distal dendritic compartments. Dendrodendritic excitatory inputs are largely absent in truncated GCs because these synapses are found primarily in the EPL (Price and Powell, 1970a,b). A second ben- 
efit is that we can exploit the diversity in apical and basal dendritic arborization extent within our population of truncated GCs to ask whether specific types of intrinsic active currents and synaptic inputs are enriched in different dendritic regions of GCs.

The comparative approach used in our study was inspired by the classic Sanger sequencing method in which nucleotide sequences were deduced by comparing DNA fragments of different length (Sanger et al., 1977). Our analysis of truncated GCs, described below, resulted in three major findings about the specialization of GC dendrites: (1) the importance of the basal dendritic arbor as a second frequent target of excitatory synaptic input, (2) the central role the proximal apical dendrite plays in initiating APs, and (3) $\mathrm{LVA} \mathrm{Ca}^{2+}$ current is also expressed in the proximal apical dendrite and functions to accelerate $\mathrm{Na}^{+}$spike firing. We then used additional experimental manipulations to verify the correlations related to AP initiation sites and the origin of lowthreshold spikes and then define the likely functional impact of proximal and distal excitatory synaptic inputs.

In truncated GCs, the extent of the apical dendrite was reduced from $336.6 \pm 19 \mu \mathrm{m}$ in intact GCs $(N=23)$ to $78.0 \pm 5.8$ $\mu \mathrm{m}$ in truncated GCs (range $11-212 \mu \mathrm{m} ; N=68$ ). Truncated GCs appeared to preserve much of the normal variability in basal dendrite number and extent described previously in mature GCs. We confirmed this by analyzing the basal dendrite extent and variability in reconstructions published in four previous studies (Price and Powell,1970a; Schneider and Macrides, 1978; Matsutani and Yamamoto, 2004; Burton and Urban, 2015). We found in these published reconstructions a similar mean length of the longest basal dendrite $(48.9 \mu \mathrm{m} ; N=21 \mathrm{GCs})$ as our population of GCs $(70.0 \mu \mathrm{m} ; N=67)$ as well as similar levels of cell-to-cell variability in basal dendrite length $(\mathrm{CV}=0.46$ in previous studies vs 0.50 in our truncated GCs). The trend toward slightly smaller basal dendrite lengths in prior work, all using fixed OB sections, may be attributable to tissue shrinkage during fixation. The number of basal dendrites did not differ between truncated GCs $(1.7 \pm 0.1 ; N=67)$ and GCs with intact apical dendrites recorded in this study $(2.0 \pm 0.2 ; N=20 ; p>0.05)$. There was no difference in the mean depth within the GCL of GC somata in intact $(126.3 \pm 12.6 \mu \mathrm{m}$ below the MCL) and truncated GCs (143.2 \pm $7.7 \mu \mathrm{m} ; p>0.05, T=1.168$; unpaired $t$ test). Because GCs continue to be generated postnatally (Petreanu and AlvarezBuylla, 2002; Carleton et al., 2003; Matsutani and Yamamoto, 2004; Whitman and Greer, 2007; Kelsch et al., 2008), different developmental stages likely contributes to the natural variation in basal dendrite arborization though all GCs included in this study are "mature" by standard electrophysiological benchmarks (e.g., firing repetitive APs; Carleton et al., 2003).

As illustrated in Figure $1 B$, the electrophysiology of truncated GCs showed little evidence of damage due to the severed dendrite, which presumably sealed over, as demonstrated in previous recordings of isolated hippocampal dendritic segments (Kavalali et al., 1997). Both intact and truncated GCs could fire repetitively in response to depolarizing steps. The input resistance assayed by hyperpolarizing steps was significantly higher in truncated GCs than intact GCs (Fig. 1C); the opposite of what would be expected if dendrite severing resulted in a continuous electrical leak to the extracellular solution. When sorted by their apical dendrite length (Fig. 1D), we found only a weak negative correlation ( $\mathrm{R}$ $\sim-0.3$ ) between apical length and input resistance in truncated GCs. By contrast, sorting truncated GCs by the extent of their basal dendritic arbor generated a robust and statistically significant negative correlation $(\mathrm{R} \sim-0.46$; Fig. $1 E)$ with input resistance. Presumably active conductances (especially leak $\mathrm{K}^{+}$current active near rest) are distributed throughout the basal dendrites, reducing the input resistance of truncated GCs with very long basal dendrites or more primary basal dendrites. There was no statistically significant difference in the bias current required to maintain intact and truncated GCs at a holding potential of -70 $\mathrm{mV}(p>0.05 ; T=0.935$; unpaired $t$ test $)$. The negative correlation between basal dendrite extent and input resistance in truncated GCs was not evident in our population of intact GCs $(r=$ $0.09 ; p>0.05)$, suggesting that the high spontaneous synaptic tone present in intact GCs decreased the apparent input resistance and masked the presence of active $\mathrm{K}^{+}$channels distributed along the basal dendritic arbor.

\section{Synaptic inputs to basal and proximal dendrites}

Spontaneous EPSPs, though greatly reduced in frequency, were evident in nearly all truncated GCs tested (spontaneous EPSPs present in 67/71 truncated GCs). In most truncated GCs, the spontaneous EPSPs remaining when the distal apical dendrite was removed had large amplitudes $(>1 \mathrm{mV})$ and fast rising phase kinetics compared with DD EPSPs (Pressler and Strowbridge, 2017). The properties of these EPSPs are described in a parallel study from our group that also exploited truncated GCs. Spontaneous IPSPs were minimized in our experiments by holding GCs at $-70 \mathrm{mV}$, near the $\mathrm{GABA}_{\mathrm{A}}$ receptor potential, precluding analysis of IPSP properties and frequency.

Across our population of 71 truncated GCs, the highest frequency of spontaneous EPSPs was $2.8 \mathrm{~Hz}$ (in a GC with $67 \mu \mathrm{m}$ apical dendrite and $196 \mu \mathrm{m}$ basal dendrite extent). The mean EPSP frequency was $0.60 \pm 0.07 \mathrm{~Hz}$ ). As shown in the two histograms in Figure $2 B$ (with logarithmic time axes), this maximal rate of spontaneous EPSPs in truncated GCs is far lower than we observed in a majority of intact GCs (73\% of intact GCs had higher frequencies of spontaneous EPSPs). The large difference in mean spontaneous EPSP rates in intact (11.4 Hz; Fig. 1C) and truncated GCs supports the hypothesis that distal DD EPSPs dominate the synaptic input impinging on intact GCs.

Subdividing our population of truncated GCs based on apical dendrite length failed to reveal statistically significant differences in the rate of spontaneous EPSPs $(p>0.05 ; T=1.354$; unpaired $t$ test; comparison based on above/below median apical dendrite length, $65 \mu \mathrm{m})$. However, a parallel division of truncated GCs based on the extent of the basal dendrite revealed $\sim$ twofold more frequent spontaneous EPSPs in GCs with more extensive basal dendritic arbors (Fig. 2D; median basal dendritic extent $=137$ $\mu \mathrm{m})$. We also assayed the correlation between dendritic extent and synaptic tone in our population of truncated GCs to determine whether excitatory synapses were more abundant on the proximal apical or basal dendrites. As shown in Figure $2 E$, there was little correlation between the rate of spontaneous EPSPs and the extent of the apical dendrite $(r=0.07 ; p>0.05)$. By contrast, we found a statistically significant correlation between EPSP rate and the extent of the basal dendrite $(r=0.44$; Fig. $2 F)$. This correlation between spontaneous EPSP rate and the basal dendritic extent was higher than the EPSP rate correlation with the total dendritic length (apical and basal) measured in the same cells $(r=0.22)$, providing additional evidence that the basal dendritic arbor is a frequent target of excitatory synaptic inputs to GCs.

\section{Action potentials in granule cells}

Although truncated GCs generated large-amplitude APs (Fig. $1 B)$, the mean amplitude of APs in truncated GCs tended to be slightly $(\sim 2.5 \mathrm{mV})$ smaller than APs evoked under similar con- 
A
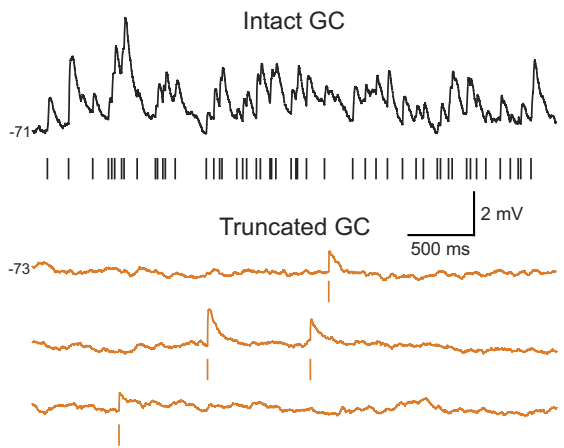

B
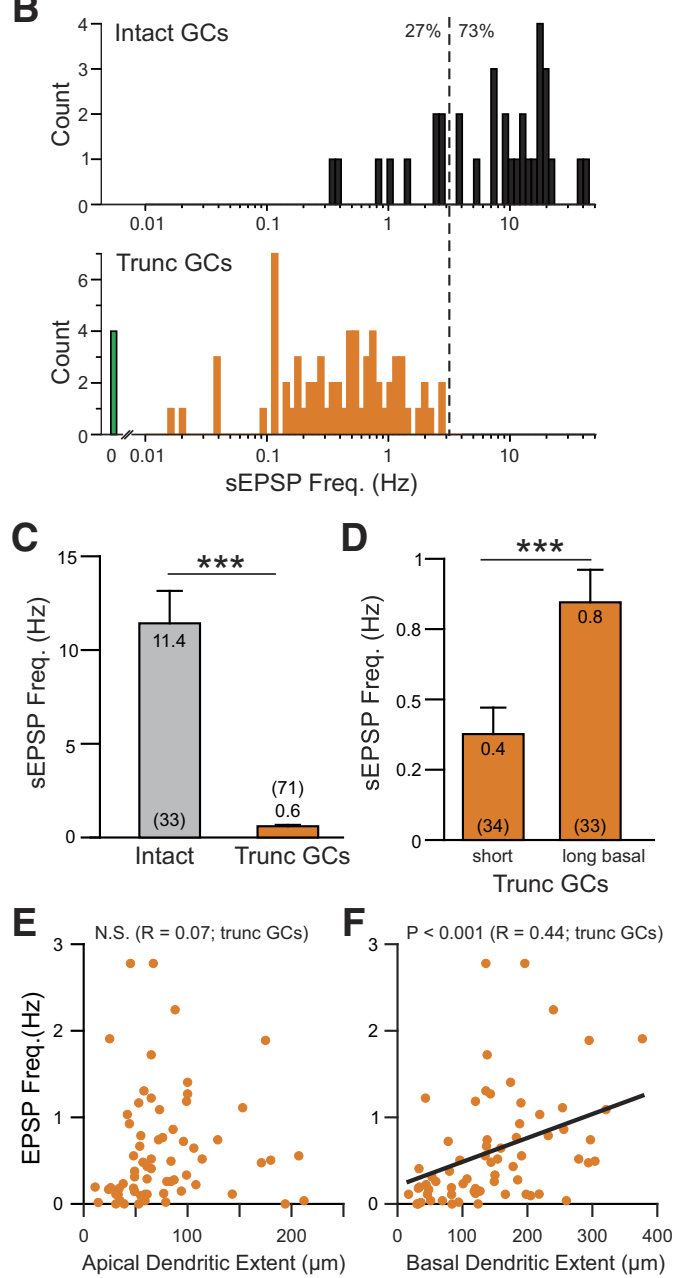

Figure 2. Spontaneous EPSPs in intact and truncated GCS. A, Example voltage records illustrating frequent spontaneous EPSPs in an intact GC (top, black traces) and a truncated GC with infrequent spontaneous EPSPs (bottom, orange traces). Vertical lines indicate onset times of SEPSPs detected automatically. The time and voltage calibration are the same in both sets of traces. $\boldsymbol{B}$, Summary histograms of sEPSP frequency in 33 intact GCS (top) and 71 truncated GCS (bottom). Event frequency indicated on $\log$ (base10) scale. Green bar indicates the number of truncated GCS with no detected sEPSPs. Vertical dashed line denotes highest sEPSP frequency detected in population of truncated GCS. C, Plot of SEPSP frequency in intact and truncated GCs; percentage of intact $\mathrm{GCS}$ above and below that $\mathrm{SEPSP}$ frequency indicated next to dashed line. ${ }^{* * *} p=5.133 \mathrm{E}-15, T=9.050$, unpaired $t$ test. $\boldsymbol{D}$, Plot of sEPSP frequency in truncated GCS with short (less than median extent, $137 \mu \mathrm{m}$ ) and long (greater than median extent) basal dendrite arbors. ${ }^{* * *} p=0.00246, T=3.151$, unpaired $t$ test. $E$, Plot of $s E P S P$ frequency versus apical dendrite extent in 67 truncated GCs. Linear regression was not statistically significant. $\boldsymbol{F}$, Plot of sEPSP frequency versus basal dendrite extent in the same group of truncated GCs. Linear regression is statistically significant (fit parameters indicated in illustration). ditions in intact GCs (Fig. 3A, B; difference not statistically significant, $p>0.05)$. This small average amplitude difference, however, masked larger differences among subpopulations of truncated GCs: those with apical dendrites greater than the median length of all truncated GCs had $\sim 7 \mathrm{mV}$ larger AP amplitudes than truncated GCs with short apical dendrites (Fig. 3B). When truncated GCs were subdivided into smaller groups based on median apical dendrite length, only truncated GCs with long apical dendrites had mean AP amplitudes as large as in intact GCs (Fig. 3B, right bars). The amplitude of APs in truncated GCs with the shortest apical dendrites were $\sim 10 \mathrm{mV}$ lower than APs in both intact GCs and truncated GCs with long $(>80 \mu \mathrm{m})$ apical dendrites. Dividing truncated GCs based on the total extent of the basal dendrites (greater or less than the median values) failed to reveal significant differences in AP amplitude $(p>0.05, T=$ 1.668 for total basal extent). These results indicate that voltagegated $\mathrm{Na}^{+}$channels located in the proximal apical dendrite, at least through the initial $80 \mu \mathrm{m}$, are required to generate fullheight APs.

APs evoked in truncated GCs had a longer mean duration than APs evoked in intact GCs (by $\sim 0.3 \mathrm{~ms}$; Fig. $3 C$ ). Within our population of truncated GCs, cells with longer apical dendrites (greater than the median apical length) had significantly narrower APs than truncated GCs with short apical dendrites (Fig. $3 C)$. There was no significant difference in AP width in truncated GCs grouped by total basal dendritic extent $(p>0.05 ; T=0.0336$ for above/below median basal dendritic extent), providing additional evidence that the apical dendrite is more important for AP generation than the basal dendritic arbor.

The difference in AP amplitude in truncated GCs with long and short apical dendrites suggests that voltage-gated $\mathrm{Na}^{+}$channels responsible for driving the rising phase of the AP are likely concentrated in the proximal segment of the apical dendrite. We tested this hypothesis by computing the maximal slope of the AP rising phase which provides a more direct measure of $\mathrm{Na}^{+}$density than AP amplitude (Sun et al., 2014; Losonczy et al., 2008). In intact GCs and truncated GCs with long apical dendrites, the membrane potential versus $V_{\mathrm{m}}$ slope plot followed overlapping trajectories (Fig. 3D) suggesting that APs in both cell populations were driven by similar constellations of voltage gated $\mathrm{Na}^{+}$channels. This similarity implies that $\mathrm{Na}^{+}$channels located in the distal apical dendrite, although likely necessary to support active spike propagation (Egger et al., 2003, 2005; Egger, 2008; Zelles et al., 2006), do not contribute significantly to the AP recorded in the GC somata. In truncated GCs with short apical dendrites, by contrast, the $V_{\mathrm{m}}$ versus $V_{\mathrm{m}}$ slope relationship followed a different trajectory and reached a lower maximal slope than in intact GCs (Fig. 3D, orange plot). Over our population of GCs, the maximal $V_{\mathrm{m}}$ slope reached during the AP in truncated GCs with short apical dendrites (less than the median extent) was significantly lower than both intact and truncated GCs with long apical dendrites. The maximal $V_{\mathrm{m}}$ slope was nearly identical in intact and GCs and truncated GCs with long apical dendrites (89 vs 85.5 $\mathrm{V} / \mathrm{s}$ ). In a parallel analysis, we found no significant difference in the AP maximal slope in truncated GCs subdivided by their basal dendritic extent (above and below the median extent; $p>0.05$; $T=0.297)$. These results confirm that full-height somaticallyrecorded APs require $\mathrm{Na}^{+}$channels located proximal apical dendrite (through the first $\sim 80 \mu \mathrm{m}$ ). The differences in AP amplitude and kinetics associated with longer apical dendrites did not affect AP threshold which was similar across truncated GCs with long and short apical dendrites (also both nearly identical to the threshold recorded in APs in intact GCs; Fig. 3F). 


\section{A}
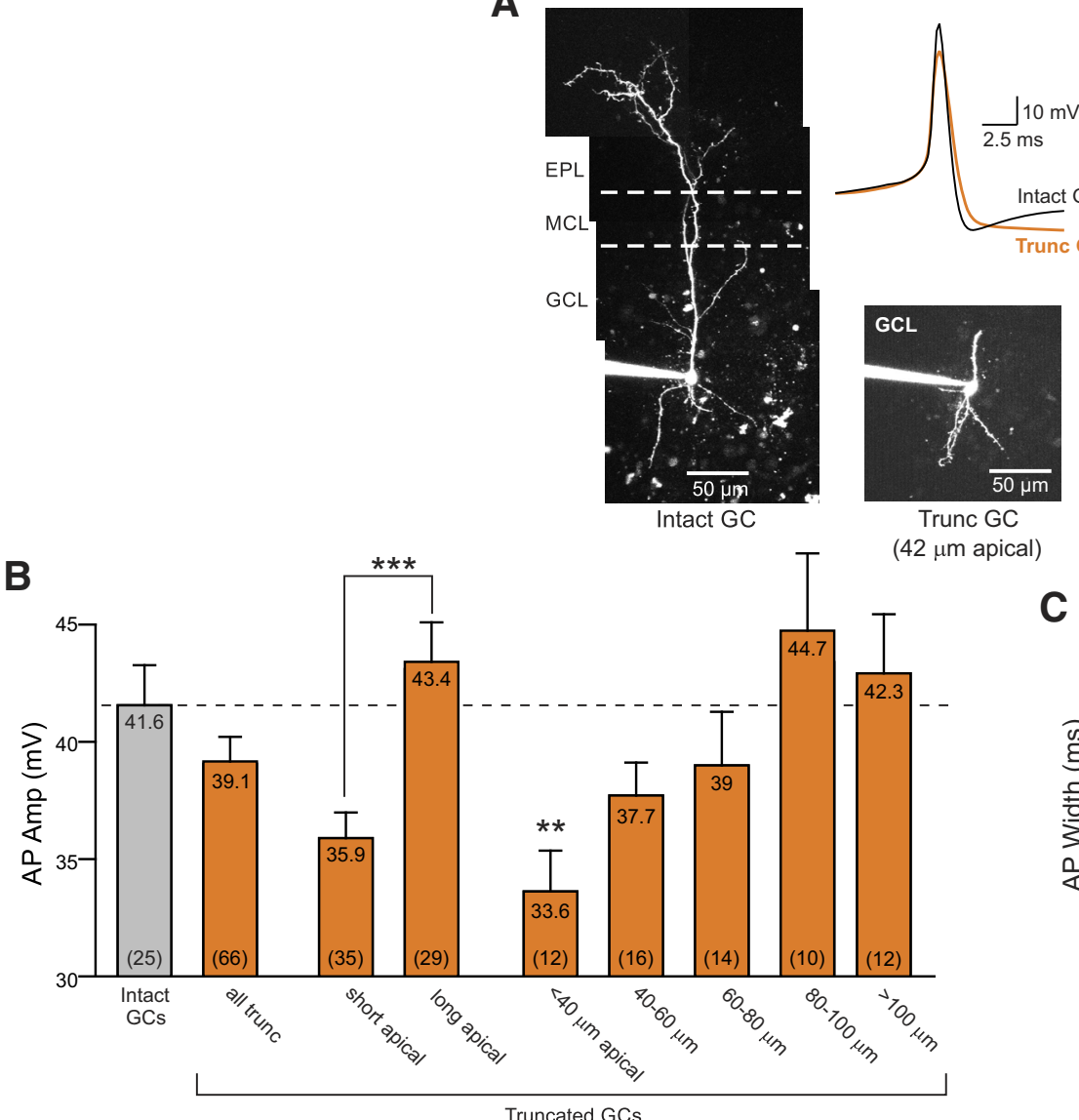

Trunc GC

(42 $\mu \mathrm{m}$ apical)

D

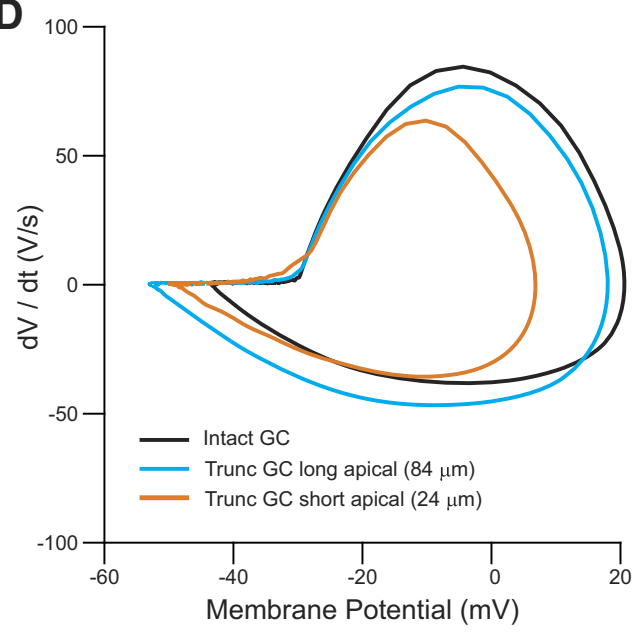

E

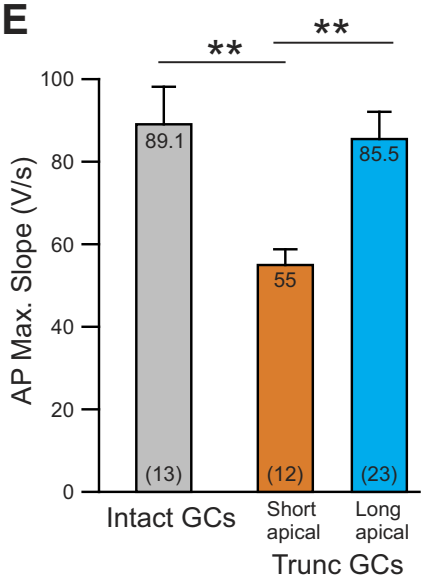

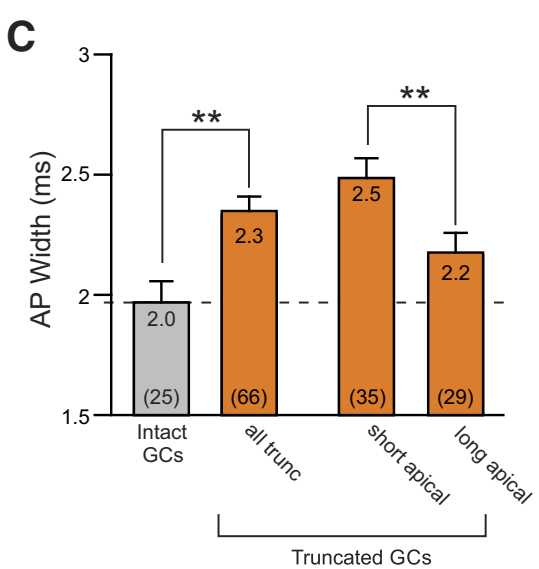

$\mathbf{F}$

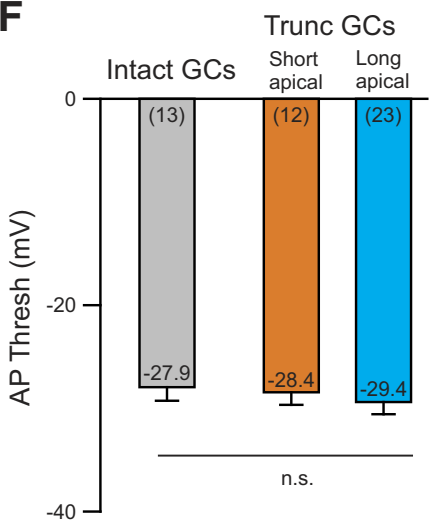

Figure 3. Smaller AP amplitudes and rising-phase slopes in truncated GCs with short apical dendrites. A, Example APs from intact (black trace) GC and a truncated GC with short apical dendrite (42 $\mu \mathrm{m}$; orange trace). Reconstructions of both cells (left, intact GC; bottom traces, truncated GC). $\boldsymbol{B}$, Plot of AP amplitude (threshold to peak) in intact GCs (gray bar) and different subsets of truncated $\mathrm{GCs}$ (orange bars). Short/long apical relative to median apical length, $65 \mu \mathrm{m}$ : ${ }^{* * *} p=2.924 \mathrm{E}-4, T=3.839$, unpaired $t$ test; different from intact, $<40 \mu \mathrm{m}$ apical: ${ }^{* *} p=0.020, T=2.812$, unpaired test. C, Plot of AP width measured at threshold in intact (gray) and subgroups of truncated GCs (orange bars). Intact/all truncated: ${ }^{* *} p=7.915 \mathrm{E}-4, T=3.259$, unpaired $t$ test; short/long apical: ${ }^{* *} p=0.011, T=2.618$, unpaired $t$ test. $D$, Plot of rising phase slope versus membrane potential for one AP from an intact GC (black curve), a truncated GC with a long apical dendrite (blue) and a truncated GC with a short apical dendrite (orange). $\boldsymbol{E}$, Plot of maximum AP rising phase slope in three groups of $\mathrm{GCs}$. Intact/short apical, less than median length: ${ }^{* *} p=0.0057, T=3.213$; short/long apical, greater than median length: ${ }^{* *} p=0.0050, T=3.168$. Both unpaired $t$ tests. $\boldsymbol{F}$, Plot of AP threshold in the three groups of GCs shown in $\boldsymbol{E}$. n.s. $=$ not significant.

The preceding analysis suggests that in intact GCs, APs likely originate in the proximal $80 \mu \mathrm{m}$ of the apical dendrite because only truncated GCs with apical dendrites $>80 \mu \mathrm{m}$ had full-height APs. We used two approaches to test this hypothesis. First, we used two-photon $\mathrm{Ca}^{2+}$ photometry in intact GCs filled with the $\mathrm{Ca}^{2+}$ indicator dye Cal520 to assay the amplitude and kinetics of AP-triggered intracellular $\mathrm{Ca}^{2+}$ accumulations. In this method, very small amplitude sinusoidal waveforms are applied to the $\mathrm{X}$ and $\mathrm{Y}$ galvanometer mirrors that control the laser beam position, creating small diameter circular trajectories that could be centered on different dendritic segments (see Materials and Methods). Because fluorescence intensity is sampled continuously in one region rather than periodically during line or raster scans, this approach enables high signal-to-noise ratios (SNRs) and ex- 
cellent temporal resolution of $\mathrm{Ca}^{2+}$ indicator responses. With the bandwidth of the fluorescence detection system reduced from $\sim 1 \mathrm{MHz}$ used with raster scanning to $\sim 1 \mathrm{kHz}$ in these experiments, the SNR of $\mathrm{Ca}^{2+}$ transients generated by APs detected photometrically in individual experiments often exceeded 40 (Fig. $4 A$ ). To our knowledge, this is the first report of experimental results obtained two-photon circling $\mathrm{Ca}^{2+}$ photometry though several reports used single-photon confocal photometry with the laser beam parked in one position (Escobar et al., 1994; DiGregorio et al., 1999; Nakamura et al., 2015).

We initially used two-photon $\mathrm{Ca}^{2+}$ photometry to define the fractional change in Cal520 fluorescence elicited by APs in intact GCs at different points along the dendritic arbor. As shown in the example spike-aligned traces in Figure $4 B$ and the summary plot in $4 C$, the magnitude of the AP-evoked $\mathrm{Ca}^{2+}$ transient (expressed as $\Delta F / F)$ increased by $>$ twofold when the photometry spot was moved from proximal $(10-25 \mu \mathrm{m})$ to distal $(150-200 \mu \mathrm{m})$ sites along the apical dendrite. The magnitude of the spike-evoked $\mathrm{Ca}^{2+}$ transients was similar in the basal dendrites and the most proximal apical dendritic region tested. The same pattern of larger $\Delta F / F$ responses in more distal apical dendritic compartments found in the summary plot (Fig. 4C) was also evident in individual experiments $(4 B)$.

Increasing $\Delta F / \mathrm{FCa}^{2+}$ indicator responses at more distal dendritic sites are commonly observed in a variety of cell types and may reflect increasing surface-to-volume ratios as the dendritic arbor tapers (Kullmann and Kandler, 2008) as well as potentially different densities and subtypes of $\mathrm{Ca}^{2+}$ channels in different dendritic compartments. We used voltage-clamp step depolarizations in a second series of intact GC recordings to ask whether different distributions of voltage-gated $\mathrm{Ca}^{2+}$ channels were responsible for the larger $\Delta F / F$ responses in distal dendrites. As shown in Figure $4 D$, the fractional change in Cal520 fluorescence evoked by $5 \mathrm{~ms}$ duration steps to $+10 \mathrm{mV}$ was similar across the same set of dendrite locations studied in current-clamp experiments. This result indicates that the lower fractional change in Cal520 fluorescence evoked in proximal apical and basal dendrites by APs was not because of lower $\mathrm{Ca}^{2+}$ channel densities in these compartments. Although we $\operatorname{did}$ not assay $\mathrm{Ca}^{2+}$ responses to different amplitude voltage-clamp steps in this study, the relatively brief duration of the voltage-clamp pulses used biases these $\mathrm{Ca}^{2+}$ transients to reflect primarily high-threshold channels and minimizes the contribution from LVA Ca ${ }^{2+}$ channels. In four experiments we confirmed that the $\mathrm{Ca}^{2+}$ indicator response evoked by $5 \mathrm{~ms}$ steps to $+10 \mathrm{mV}$ was unaffected by $50 \mathrm{~ms}$ prepulses to $-45 \mathrm{mV}$, suggesting that response to the $5 \mathrm{~ms}$ pulse was primarily mediated by high-threshold $\mathrm{Ca}^{2+}$ channels $(\Delta F / F$ $94.0 \pm 6.2 \%$ of control step responses to $+10 \mathrm{mV}$ without prepulses; $p>0.05, T=0.960$, paired $t$ test).

We next used GC AP waveforms as voltage-clamp command signals to determine whether the $\mathrm{Ca}^{2+}$ transients recorded in distal GCs dendrites arose from active spike propagation. As shown in Figure $4 E$, we found the largest fractional change in Cal520 florescence responses to AP waveform stimuli in the most proximal apical dendritic compartment. Calcium elevations associated with voltage-clamp AP waveforms then decremented when tested at more distal apical dendritic locations. The opposite spatial pattern of $\Delta F / F$ responses along the apical dendrite to physiological APs (recorded under current-clamp conditions; Fig. $4 C$ ) and voltage-clamped AP waveforms (4E) suggests that AP propagation relies on active conductances distributed along the apical dendrite to invade the more distal regions of the apical arbor.
The $\Delta F / F$ magnitude changes in photometry experiments suggest that GCs use active conductances to enable APs to propagate into the distal apical dendrite. The kinetics of the AP-evoked Cal520 fluorescent transient also varied at different locations. Instead of continuously increasing with distance from the soma, the rising phase kinetics of the photometry signal was more rapid in the mid apical dendrite (50-100 $\mu \mathrm{m}$ from the soma) and then slowed at more distal apical dendritic compartments (Fig. $4 F$ ). The rising phase kinetics of the $\Delta F / F$ response were significantly faster in the mid-apical zone than in either very proximal or more distal apical zones. We found no difference in the rising phase kinetics in parallel experiments with voltage-clamp steps to +10 $\mathrm{mV}$ (5-50\% rise times of $\Delta F / F$ signal: $2.45 \pm 0.17 \mathrm{~ms}$ for apical $10-25 \mu \mathrm{m}, 2.15 \pm 0.11 \mathrm{~ms}$ for apical $50-100 \mu \mathrm{m}, 2.05 \pm 0.24 \mathrm{~ms}$ for apical 150-200 $\mu \mathrm{m}$; means not different, all $p>0.05$ ), suggesting that kinetic difference in AP-evoked transients did not reflect differences in voltage-dependent $\mathrm{Ca}^{2+}$ channel expression or properties in different dendritic regions. The difference in kinetics may reflect a higher density of voltage-gated $\mathrm{Na}^{+}$channels in the mid-apical dendrite, as suggested by a requirement for apical dendrites $>80 \mu \mathrm{m}$ to generate full-height APs in our analysis of truncated GCs subgroups (Fig. 3B). Although indirect, the similar findings in these different experiments suggest that in intact GCs, APs likely originate in the mid-apical zone, $50-100 \mu \mathrm{m}$ from the soma.

In the second approach to define the functional properties of the proximal apical dendrite, we used focal application of the voltage-gated $\mathrm{Na}^{+}$channel blocker TTX to confirm the central role for the proximal apical dendrite spike generation. We visualized the cell body and apical dendrite in 29 intact GCs to guide focal TTX application under live two-photon imaging (Fig. 5A). In Figure 5, we compared the effectiveness of focal TTX in modulating the spike discharge during standardized responses (250 ms current steps that evoked 3-5 APs). Only TTX applied to the proximal apical dendrite (10-100 $\mu \mathrm{m}$ from cell body) reduced the number of APs evoked by the test step (Fig. $5 B$; reduction in number of APs in 8/27 experiments). In three experiments, TTX applied to the proximal apical dendrite blocked all $\mathrm{Na}^{+}$ spikes (Fig. 5B, example with recovery following TTX washout). In other five successful experiments, TTX applied to the proximal apical dendrite reduced the number of APs evoked by the step stimulus by an average of $46 \pm 5.8 \%$. In the 7 of 8 successful experiments with proximal apical dendrite TTX, the effect of TTX pressure application reversed within $2 \mathrm{~min}$, as illustrated in Figure $5 B$. (The eighth experiment showed only a partial recovery.)

Similar applications of TTX to the soma $(N=7$; Fig. $5 C)$, distal apical dendrite ( $>150 \mu \mathrm{m}$ from cell body; $N=7$; Fig. $5 D$ ) and basal dendritic locations $(N=10)$ all consistently failed to alter the number of APs evoked. Results from these experiments are summarized in Figure $5 E$ and demonstrate that transient blockade of $\mathrm{Na}^{+}$channels located proximal apical dendrite reduced spike discharges initiated by somatic current injections. Focal application of TTX to the cell body not only failed to modulate the number of APs triggered by the depolarizing step but also did not affect the AP latency (Fig. $5 F$ ) or the amplitude of the initial AP ( $p>0.05$, paired $t$ test). The inability of TTX applied to the soma to modulate AP properties also provides evidence for the spatial selectivity of the pressure application method. Together with the comparative analysis of truncated GCs with 
A

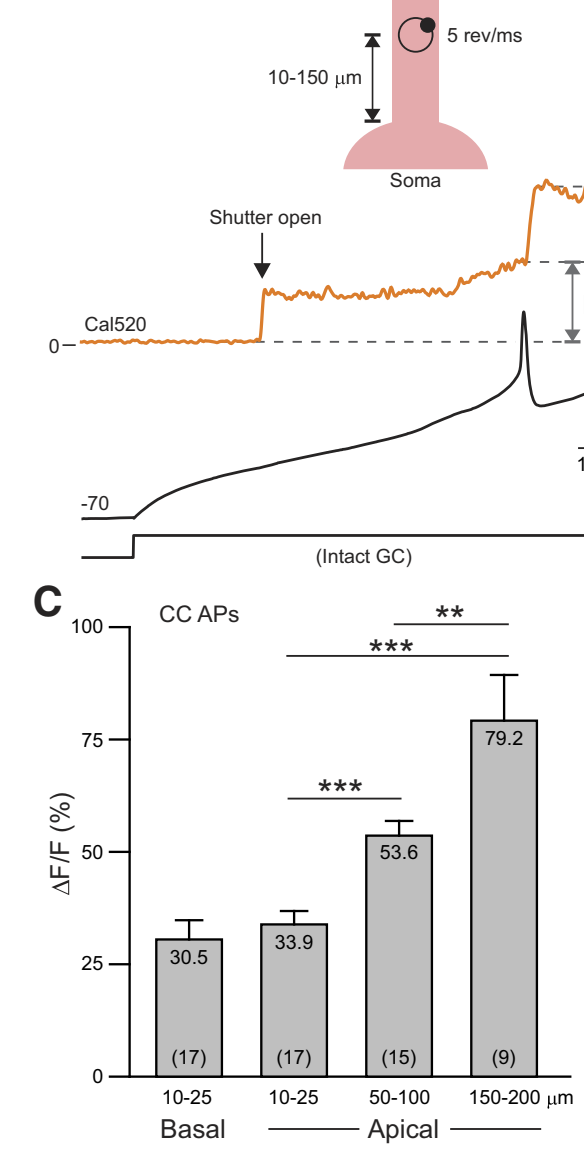

E

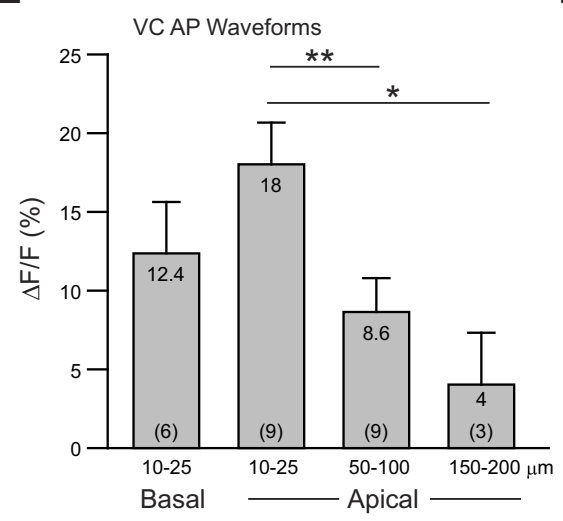

B

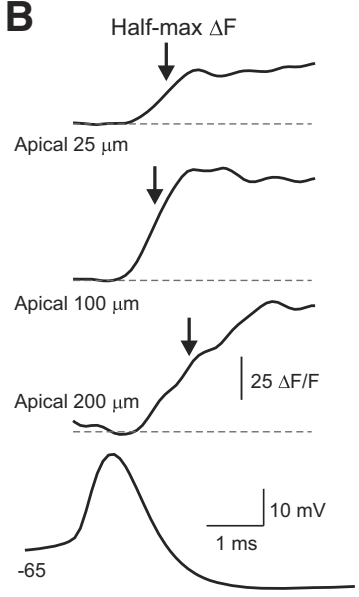

D

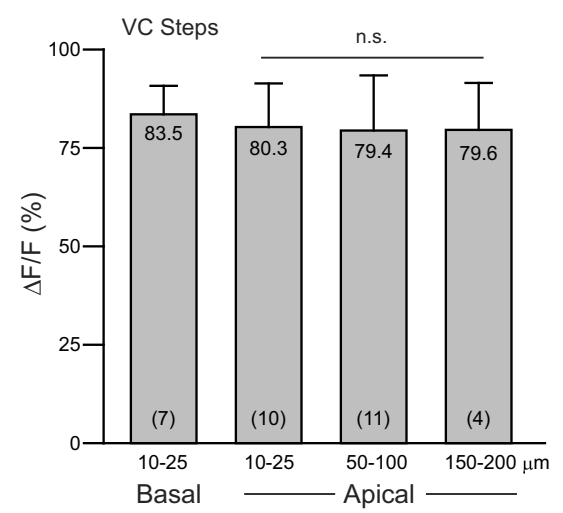

$\mathbf{F}$

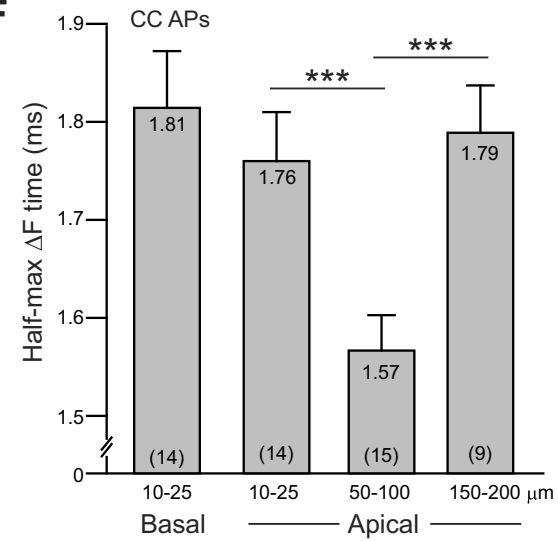

Figure 4. Optical measurement of AP triggered $\mathrm{Ca}^{2+}$ accumulation in different dendritic compartments. $A$, Example simultaneous measurement of somatic membrane potential (black trace) and Cal520 fluorescence in the apical dendrite (orange trace; 10 $\mu \mathrm{m}$ from the soma; single trial) in an intact GC. AP-triggered change in florescence indicated by vertical arrows on right $(\Delta F)$, relative to fluorescent signal immediately before AP $(\boldsymbol{F})$. See Materials and Methods for photometry details. $\boldsymbol{B}$, Example photometry responses to a single AP recorded at three different locations along the apical dendrite of the same GC. Each responses represents spike-aligned average of five trials. Vertical arrows indicate timing of half-maximal AP-evoked fluorescence increase. $C$, Plot of mean AP-evoked fluorescence increase in different dendritic regions. $N$ values represent number of dendritic location/cell trials; $10-25 / 50-100 \mu \mathrm{m}$ apical: ${ }^{* * *} p=2.278 \mathrm{E}-4, T=4.333 ; 10-25 / 150-200 \mu \mathrm{m}$ apical: ${ }^{* * *} p=4.263 \mathrm{E}-5, T=5.150$; $50-100 / 150-200 \mu \mathrm{m}$ apical: ${ }^{* *} p=0.018, T=2.740$. All unpaired $t$ tests. D, Plot of mean fluorescence transients evoked by 5 ms duration voltage-clamp steps from -70 to $+10 \mathrm{mV}$. $\boldsymbol{E}$, Plot of mean fluorescence transients evoked in response to voltageclamp AP waveform command in intact GCS. ${ }^{* *} p=0.0296, T=2.590 ;{ }^{*} p=0.0419, T=2.569$; both unpaired $t$ tests. Voltagegated $\mathrm{Na}^{+}$channels blocked using QX314 added to the internal solution in $\boldsymbol{D}$ and $\boldsymbol{E}$. F, Plot of timing of half-maximal AP-evoked fluorescence transient, as illustrated in $\boldsymbol{B}$, for the same dendritic subregions. Timing relative to AP onset. $y$-Axis broken to illustrate relative differences in mean times. Ten to $25 / 50-100 \mu \mathrm{m}$ apical: ${ }^{* * *} p=0.00525, T=3.200 ; 50-100 / 150-200$ $\mu \mathrm{m}$ apical: ${ }^{* *} p=2.629 \mathrm{E}-3, T=3.559$; both unpaired $t$ tests. n.s. $=$ not significant. different length apical dendrites (Fig. 3) and the analysis of spike-evoked $\mathrm{Ca}^{2+}$ transient kinetics (Fig. $4 F$ ), these results suggest that the proximal apical dendrite is likely the initiation site for APs in GCs.

\section{Low-threshold $\mathrm{Ca}^{2+}$ current in proximal apical dendrite accelerates GC firing}

The third major difference we found between intact and truncated GCs was a reduction in the spike latency in intact neurons. We assayed spike latency by slowing increasing the amplitude of a $1 \mathrm{~s}$ duration current step until a single AP was triggered from a holding potential of -70 $\mathrm{mV}$. In intact GCs, the AP occurred with only a short $\sim 200 \mathrm{~ms}$ latency while in truncated GCs with short apical dendrites, the latency was significantly longer $(>500$ ms; Fig. 6A). In many truncated GCs with short apical dendrites, the membrane potential reached a quasi-steady-state voltage after $100 \mathrm{~ms}$ and then began to slowly depolarize before finally triggering a spike (Fig. 6A, middle, blue trace). Increasing the stimulus amplitude accelerated firing in these truncated GCs. However, even with steps that triggered two or more APs, the initial spike latency was still delayed relative to intact GCs (initial spike latency in truncated GCs firing two or more spikes $=370.1 \pm 47.3$ vs $209.4 \pm 21.6$ first spike latency in intact GCs; $p=0.0034$; $\mathrm{T}=2.849$; unpaired $t$ test).

The ability of intact GCs to fire rapidly in response to step stimuli appears to reflect recruitment of a low-threshold spike (LTS) mediated by LVA $\mathrm{Ca}^{2+}$ channels. In most intact GCs and truncated GCs with long apical dendrites, depolarizing steps that were just subthreshold for triggering APs often evoked a low-threshold $\mathrm{Ca}^{2+}$ spike (Fig. $6 A$, red traces). In these GCs, the LTS response facilitated APs because the $\mathrm{Na}^{+}$spike occurred at approximately the same time as the LTS in justsubthreshold responses (Fig. 6A, blue traces). In truncated GCs with short apical dendrites, by contrast, just-subthreshold current steps typically failed to trigger a LTS and $\mathrm{Na}^{+}$spike firing was delayed when the stimulus intensity was increased to reach the AP threshold (Fig. 6A, middle). Figure $6 B$, left plot, illustrates the tight clustering of both LTS amplitude (from just-subthreshold responses) and spike latency (from just-suprathreshold responses) in intact GCs. In truncated GCs, similar analysis reveals a larger range of both LTS amplitude and spike latency. We also found a statistically significant in- 
verse correlation between LTS amplitude and spike latency (Fig. 6B, right plot), supporting the hypothesis that the LTS functioned to accelerate AP firing in GCs with long apical dendrites.

In addition to correlating with LTS amplitude, spike latency was significantly (and inversely) correlated with the extent of the apical dendrite in truncated GCs (Fig. 6C, left plot). Spike latency was not significantly correlated with basal dendritic extent in the same population truncated GCs $(r=-0.35 ; p>0.05)$. Plot of relative frequencies of different AP latencies in truncated GCs (Fig. $6 C$, right) suggested two clusters of truncated GCs: those with early and delayed firing. When divided into two populations based on the median apical dendrite length, truncated GCs with long apical dendrites (greater than the median length) had significantly faster spike latencies than truncated GCs with short apical dendrites, consistent with the correlation plot in Figure 6B. We found no significant difference in spike latency a similar subdivision based on the basal dendritic extent (longer or shorter than median basal extent; $p>0.05 ; T=$ $1.240)$.

We next asked how long an apical dendrite was required for a truncated GC to have "intact-like" properties (a LTS following just-subthreshold stimuli and short AP latencies in response to justsuprathreshold steps). Restricting the population of truncated to GCs to those with apical extents $>100 \mu \mathrm{m}$ resulted in a short mean AP latency $(\sim 180 \mathrm{~ms})$ similar to that found in intact GCs $(\sim 210 \mathrm{~ms}$; Fig. $6 D$, gray bar). Just-subthreshold steps evoked LTS responses in all truncated GCs with apical dendritic extents $>100$ $\mu \mathrm{m}$. Together, these results suggest that LVA $\mathrm{Ca}^{2+}$ current located in the proximal apical dendrite (through the first $100 \mu \mathrm{m}$ ) is critical to enable GCs to trigger a LTS that can accelerate AP firing. The LTS response is likely to be especially important in small interneurons with high-input resistance, such as GCs, that have long membrane time constants. Without such an accelerating mechanism, GCs might not reach firing threshold within the inhalation phase of the respiratory cycle.

We then asked whether the LTS response was mediated by LVA $\mathrm{Ca}^{2+}$ channels, as previous studies have identified LVA $\mathrm{Ca}^{2+}$ current-mediated responses in OB GCs (Pinato and Midtgaard, 2003, 2005; Egger et al., 2003, 2005; Inoue and Strowbridge, 2008). We first used two-photon $\mathrm{Ca}^{2+}$ photometry in the proximal apical dendrite verify that LTS voltage responses were associated with intracellular $\mathrm{Ca}^{2+}$ elevation. As shown in the example responses in Figure 6E, a subthreshold step that triggered a LTS but no APs also triggered an increase in Cal520 fluorescence. Both the LTS voltage response and the Cal520 flu-

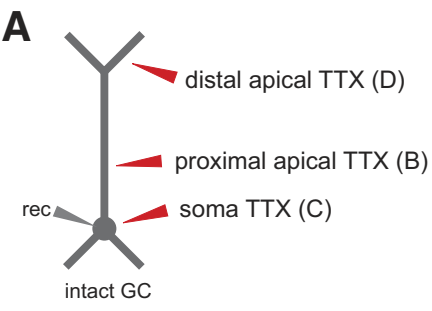

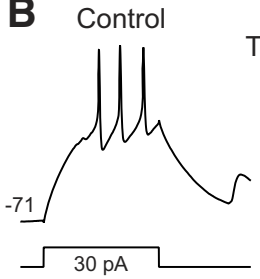

C Control

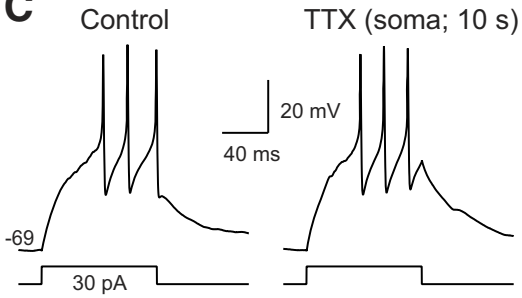

E

Figure 5. Blockade of APs by focal TTX applied to proximal apical dendrites. A, Diagram illustrating locations where TTX was applied to GCS. B, Example responses to the same current step before TTX (Control), 10, 20, and $120 \mathrm{~s}$ following a single pressure application TTX to the proximal apical dendrite, $50 \mu \mathrm{m}$ from the soma. TTX (5 $\mu \mathrm{m})$ ejected using a $300 \mathrm{~ms}, 20$ psi pressure pulse. same current step before and 10 sfollowing TTX application to the distal apical dendrite, $200 \mu \mathrm{m}$ from the soma (in the EPL).E, Plot of number of APs evoked by the same current step 10 s following focal TTX pressure application to different soma-dendritic regions. significantly different from 1: ${ }^{*} p=0.0498, T=1.897 ; 50-100$ apical: ${ }^{*} p=0.0127, T=2.582$; both two sided one-sample tests. $F$, Plot of the latency to the first AP evoked by the same current step before and $10 \mathrm{~s}$ following focal TTX application to the soma. n.s. $=$ not significant. orescence increase were blocked by the $\mathrm{LVACa}^{2+}$ channel antagonist ML218 (Fig. $6 E$, green traces). We also confirmed that nickel $(100 \mu \mathrm{M})$, another blocker of many types of LVA channel subtypes (Lee et al., 1999; Obejero-Paz et al., 2008), also blocked the LTS voltage response (Fig. $6 F$ ). Finally, we verified that LTS voltage responses could be still evoked in intact GCs with voltagegated $\mathrm{Na}^{+}$channels blocked by either bath TTX (Fig. $6 G ; N=3$ ) or intracellular QX314 (data not shown; $N=9$ ) suggesting that LVA Ca ${ }^{2+}$ channels rather than subthreshold $\mathrm{Na}^{+}$channels were likely responsible for the LTS in GCs. Both $\mathrm{Na}^{+}$channel blockers abolished step-evoked APs.

The effectiveness of ML218 in reducing the LTS-associated intracellular $\mathrm{Ca}^{2+}$ accumulation, measured photometrically, is 

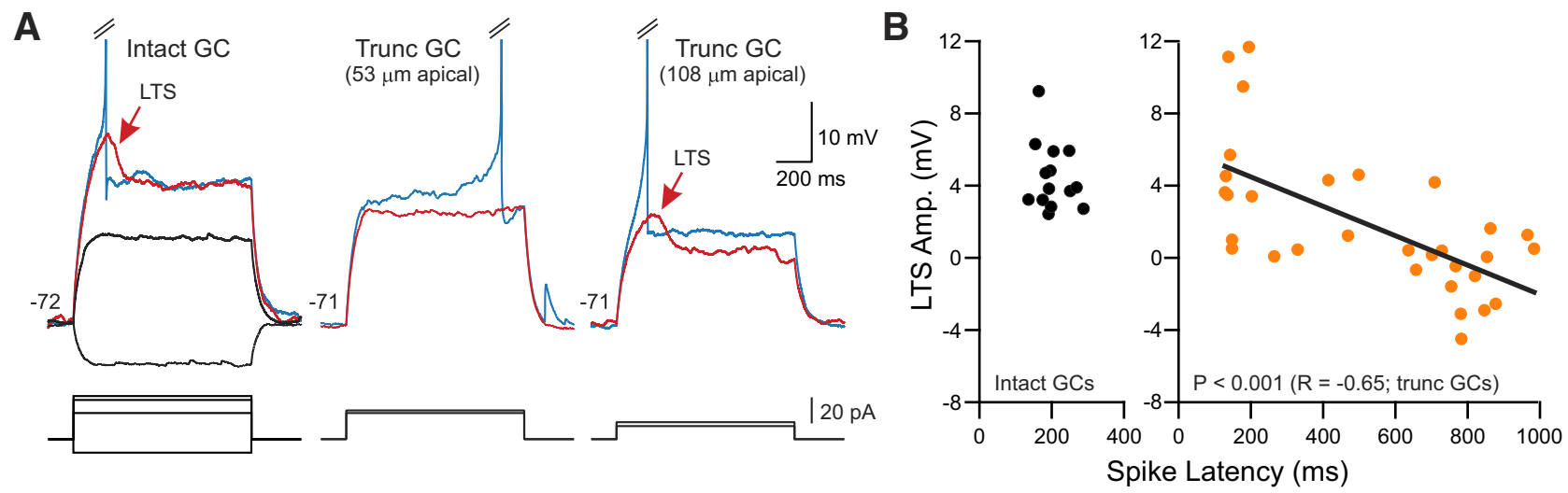

C
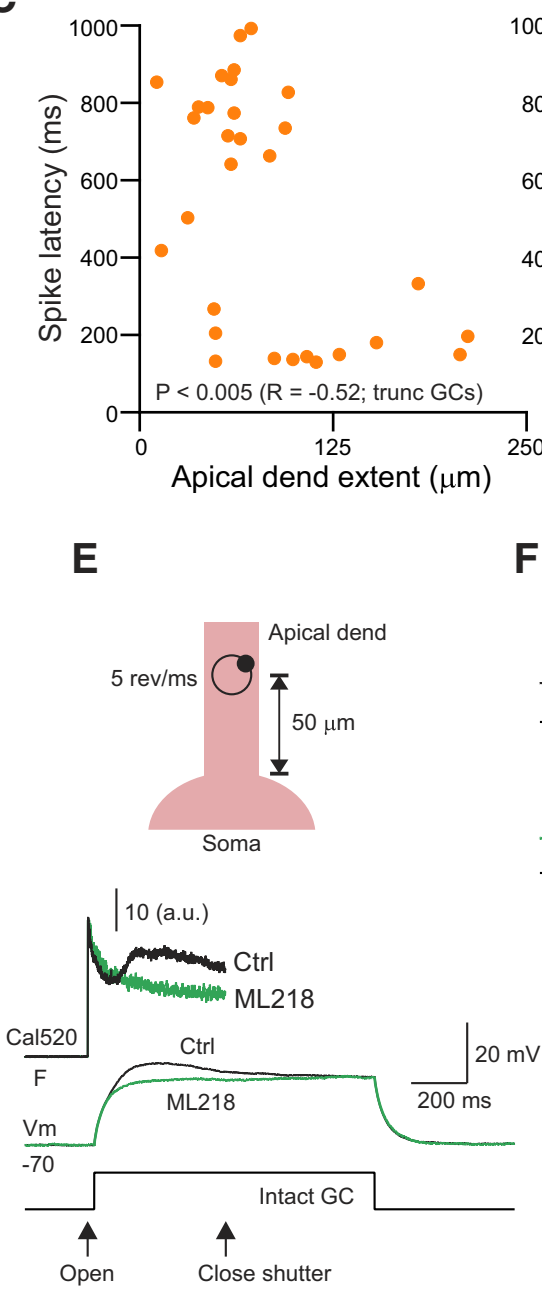

D

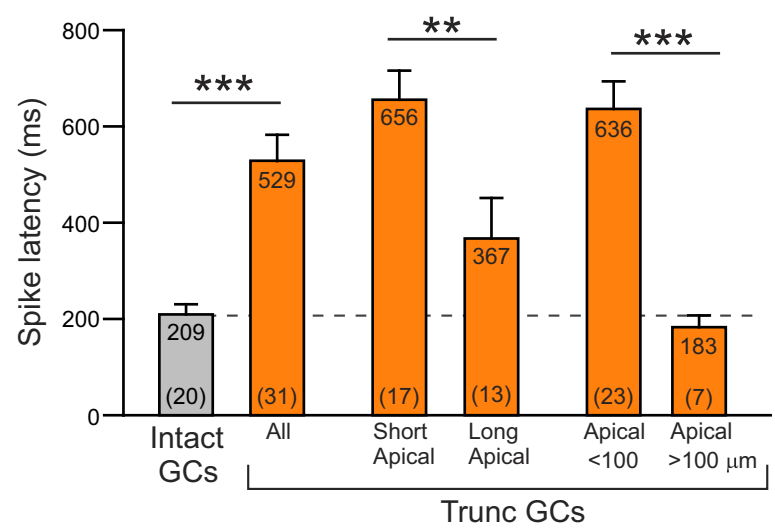

$\mathbf{F}$

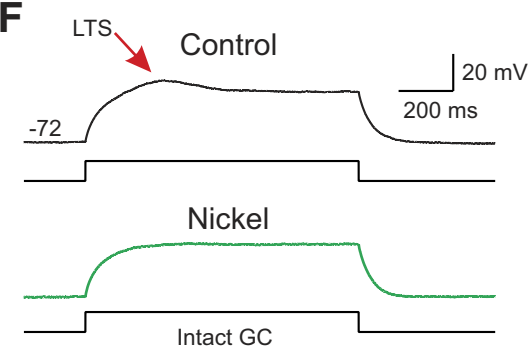

G

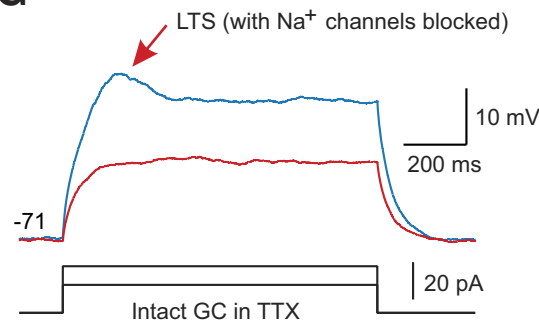

H

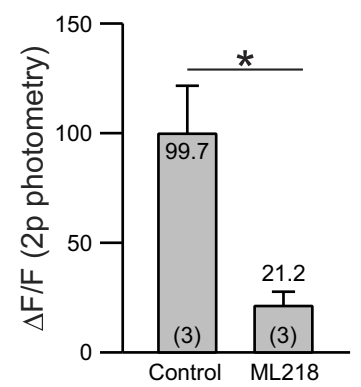

I

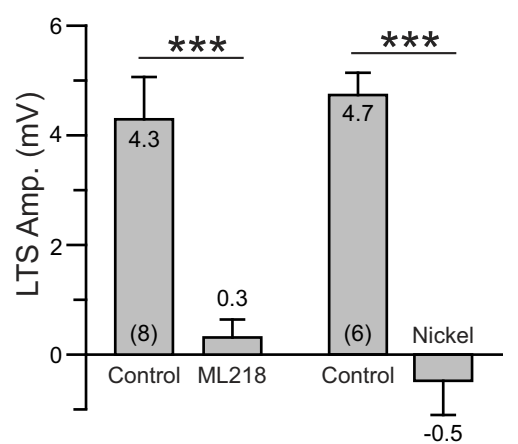

Figure 6. Low threshold $\mathrm{Ca}^{2+}$ spike requires proximal apical dendrite. $\boldsymbol{A}$, Example subthreshold (red traces) and suprathreshold (blue traces) step responses in an intact $\mathrm{GC}$ (left) and two truncated GCs (middle and right). The truncated GC with a very short apical dendrite $(53 \mu \mathrm{m})$ failed to generate a LTS with just-subthreshold steps and had a long-latency to trigger an AP with suprathreshold steps. Low-threshold spikes (red arrows) evident in responses to just-subthreshold current steps in intact GC and truncated GC with long (108 $\mu$ m; right) apical dendrite. APs truncated in $\boldsymbol{A}$. B, Plot of the relationship between AP latency and LTS amplitude in 31 truncated GCs (right, orange symbols) and 14 intact GCs (left, black). Linear regression (solid line) superposed on plot from truncated GCS. C, Summary plot of relationship between apical dendrite extent and just suprathreshold AP latency in 31 truncated GCs. Linear regression (solid line) superimposed. Histogram of spike latency in truncated GCs shown on right. $D$, Summary of spike latency in response to just-suprathreshold current steps in intact (gray bar) and truncated GCs (orange bars). .*** $p=$ $2.361 \mathrm{E}-5, T=4.464$ (intact vs all truncated); ${ }^{* *} p=0.010, T=2.758$ (long/short apical); ${ }^{* * *} p=1.271 \mathrm{E}-4, T=4.186$ (truncated GCs with apical dendrites $<0$ or $>100 \mu \mathrm{m}$ ). All comparisons tested via unpaired $t$ tests. $\boldsymbol{E}$, ML218 (1.5 $\mu$ m; green traces) also abolished the LTS response (bottom traces) and the increase in intracellular $\mathrm{Ca}^{2+}$ assayed using two-photon photometry in the proximal apical dendrite ( $50 \mu \mathrm{m}$ from the soma; top traces). $\boldsymbol{F}$, Nickel (100 $\mu \mathrm{m}$; green trace) abolished the LTS response (arrow) evoked by just subthreshold current steps in an intact GC. $\mathbf{G}$, Intact $\mathrm{GC}$ with Na ${ }^{+}$channels blocked by $1 \mu \mathrm{m}$ TTX still generates low-threshold spikes (arrow). $\boldsymbol{H}$, Summary plot of reduction in the $\mathrm{Ca}^{2+}$ increase associated with LTS by ML218 in intact GCS. ${ }^{*} p=0.0297$, $\mathrm{T}=3.920$, paired $t$ test. $I$, Plot of the reduction in LTS amplitude by ML218 (left bars) and nickel (right bars) in intact GCs. ${ }^{* * *} p=1.403 \mathrm{E}-4, T=6.687$ (ML218); ${ }^{* * *} p=0.00116, T=5.701$ (nickel), paired $t$ tests. 
A

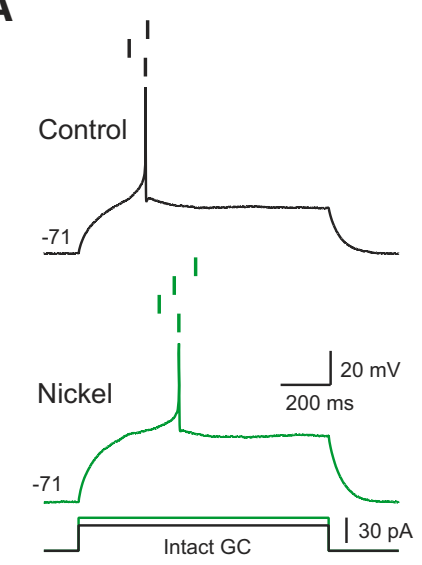

D

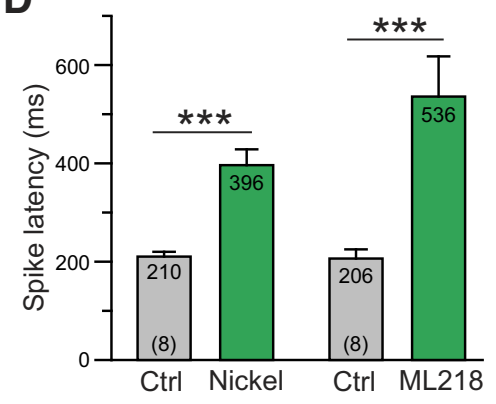

B

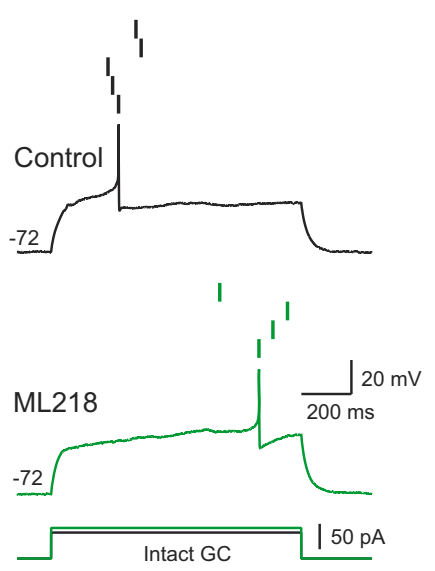

E

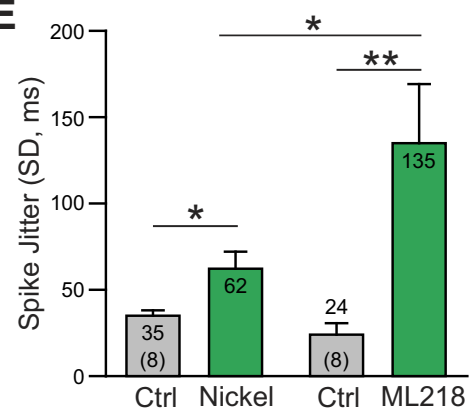

C
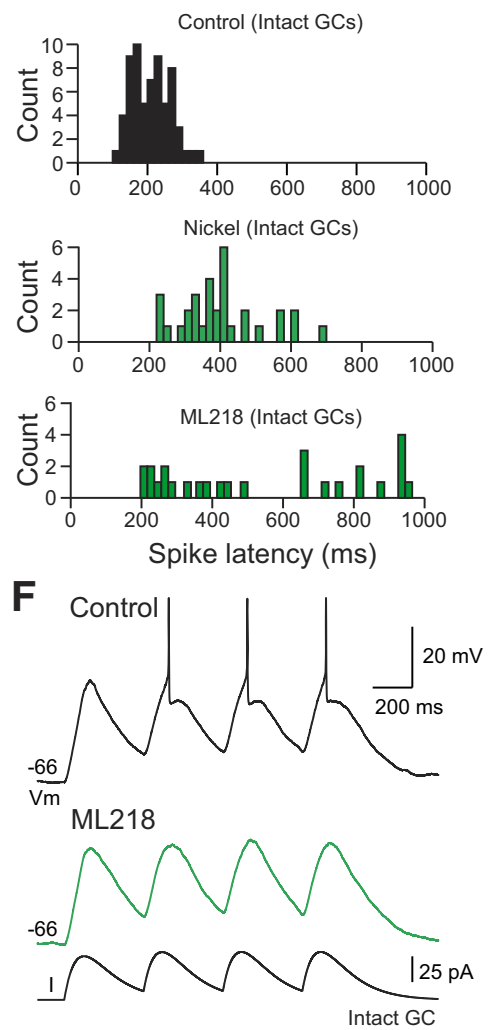

Figure 7. Low threshold $\mathrm{Ca}^{2+}$ spikes decrease spike latency and reduce jitter in GCs. A, Example responses to just-suprathreshold current steps in intact GCs before (black traces) and following attenuation of T-type $\mathrm{Ca}^{2+}$ current with nickel $(100 \mu \mathrm{M}) . \boldsymbol{B}$, Similar experiment with ML218 $(1.5 \mu \mathrm{M}) . \boldsymbol{A}, \boldsymbol{B}$, Green voltage and current traces represent just-suprathreshold trials in T-type blockers (nickel or ML218). C, Summary histograms of spike latencies in response to just-suprathreshold $1 \mathrm{~s}$ current steps under control conditions (top, black bars) and with T-type $\mathrm{Ca}^{2+}$ currents attenuated using either nickel (100 $\mu$ m; middle, green bars) or ML218 (1.5 $\mu$; bottom, green bars). All recordings from visualized intact GCs. $\boldsymbol{D}$, Plot of the mean spike latencies from the experiments presented in $\boldsymbol{A}-C^{*}{ }^{* *} p\left(\right.$ nickel) $=0.00154, T=4.418 ; * * * p(M L 218)=0.00133, T=4.543$. Both paired $t$ tests. $\boldsymbol{E}$, Plot of spike jitter (latency SD) before and after attenuation of T-type $C^{2+}{ }^{2+}$ currents with nickel or ML218. ${ }^{*} p(\mathrm{ctrl} / \mathrm{nickel})=0.0255, T=2.351$, paired $t$ test; ${ }^{* *} p(\mathrm{ctr} / \mathrm{ML} 218)=0.0107, T=2.952$, paired $t$ test; ${ }^{*} p$ (nickel/ML218) $=0.0385, T=1.909$, unpaired $t$ test. $\boldsymbol{F}$, Blocking T-type $\mathrm{Ca}^{2+}$ currents with ML218 attenuated voltage responses to phasic current waveforms that mimic rhythmic synaptic excitation during respiration (100 ms $\alpha$ functions repeated at $2.5 \mathrm{~Hz}$ ).

summarized in Figure $6 H(\Delta F / F$ reduced to $\sim 20 \%$ of control). Figure 6 I summarizes the reduction in LTS amplitude following blockade of LVA $\mathrm{Ca}^{2+}$ channels with ML218 and nickel over multiple experiments. These results demonstrate that current through LVA $\mathrm{Ca}^{2+}$ channels mediates the LTS evoked by depolarizing stimuli in GCs.

If LTS responses that accelerate spike firing are mediated by LVA Ca ${ }^{2+}$ current in the apical GC dendrite, then pharmacological blockade of LVA channels should increase AP latencies. In Figure 7 we show that two T-type LVA $\mathrm{Ca}^{2+}$ channel antagonists, nickel (7A) and ML218 (7B), do indeed increase spike latencies in intact GCs tested with just-suprathreshold current steps. Histograms of spike latencies show that both $\mathrm{LVA} \mathrm{Ca}^{2+}$ channel blockers not only increase mean spike latency but also greatly increase spike time variability. The effect of LVA blockers on mean spike timing is quantified in Figure $7 D$ and on spike timing jitter in $7 E$. These results support the hypothesis that LVA-mediated LTS responses function to oppose the tendency of interneurons with high resistance, like GCs, to have delayed firing latencies as a consequence of their long membrane time constants. In olfactory GCs, the ability to spike quickly in response of slow phasic stimuli is especially important because $\mathrm{OB}$ circuits are normally driven phasically during sniffing. Using current injections that mimic this natural input set at just-suprathreshold intensities, blockade of LVA $\mathrm{Ca}^{2+}$ channels abolishes firing (Fig. $7 F$ ). In addition to accelerating spiking in response to step stimuli, these results in- dicate that LTS responses also function to amplify the stimulus. Low-threshold spikes appear to increase the probability that GCs spike within the narrow time windows at the peaks of slow phasic depolarizing stimuli generated during sniffing.

\section{Proximal excitatory inputs trigger APs with high temporal precision}

Overall, the results of this study suggest that subregions within the GC dendritic arbor are specialized for different tasks with the proximal apical dendrite playing a critical role in both generating APs and low-threshold $\mathrm{Ca}^{2+}$ spikes that accelerate spike timing. The basal dendrite appears not to be necessary for AP generation but, our results suggest, is a largely unexplored source of excitatory input to GCs.

The proximal region of the apical dendrite we find important for AP generation overlaps with the innervation zone for centrifugal afferents to GCs arising from piriform cortex (de Olmos et al., 1978; Haberly and Price, 1978; Shipley and Adamek, 1984; Balu et al., 2007). Sensory-driven afferents, by contrast, disynaptically activate the distal apical dendrite, generating EPSPs recorded in the soma that have slower kinetics than cortical feedback EPSPs (Fig. 8A, $B$; feedback EPSPs generated by focal two-photon guided microstimulation; distal dendritic EPSPs evoked by stimulating a monosynaptically-coupled mitral cell in a paired recording from a previous study from our group; Pressler and Strowbridge, 2017). 
The faster kinetics of cortical feedback EPSPs suggests that that input could drive spike generation more efficiently than the electrotonically-slowed distal dendritic input. We used shortduration depolarizing steps that evoked voltage responses that mimicked the rising phase of cortical feedback EPSPs (Fig. 8A; time to peak $\sim 3.4 \mathrm{~ms}$ ) and distal DD EPSPs ( $8 B$; time to peak $\sim 7.2 \mathrm{~ms}$ ) to test this hypothesis. These cortical and DD responses displayed similar rise-time kinetics as the physiological synaptic inputs and could be scaled to a sufficient amplitude to reliably trigger spiking. With both 3 and $10 \mathrm{~ms}$ duration steps, we adjusted the amplitude to trigger a single AP (Fig. $8 \mathrm{C}$ ). As illustrated in Figure 8, C and D, GC spiking occurred with far less jitter when driven by cortical EPSP-like depolarizing steps than by slower DD-like steps. These results suggest that proximally-located excitatory synaptic inputs, including cortical feedback EPSP, are able to trigger more precisely-timed APs than distal DD EPSPs, reflecting both their faster kinetics and their location near the spike initiation zone.

\section{Discussion}

We make three primary conclusions in this study. First, we find that the likely site of AP generation in GCs is the proximal 100 $\mu \mathrm{m}$ of the apical dendrite. Previously, the origin of APs in these axonless interneurons was unknown. Our results also suggest that APs initiated in the proximal apical dendrite propagate actively through the distal apical dendritic arbor, based on the opposite spatial patterns of $\mathrm{Ca}^{2+}$ transient evoked by APs and AP-like voltage-clamp command signals. Our results highlight the importance of cortical feedback excitatory inputs which selectively target the same proximal apical dendritic region. Second, we find that LVA channels also located in the proximal apical dendritic region are necessary to generate low-threshold spikes that accelerate spiking in GCs. Without low-threshold $\mathrm{Ca}^{2+}$ spikes, the long membrane time constant of GCs delays spiking by $>300 \mathrm{~ms}$ in response to just-threshold stimuli, a delay that would likely reduce the probability that GCs fire during individual sniff cycles. And finally, we find the basal dendrites are an important source of excitatory input to GCs that has largely been ignored.

Together these findings support the hypothesis that GCs comprise two different functional modules: (1) the established pathway (Shepherd et al., 2007) in which sensory-driven DD excitation of distal apical GC dendrites leads to GABA release at EPL spines and (2) a novel (and motoneuron-like) synaptic integration pathway in which excitatory basalar dendritic synaptic inputs are integrated in the soma, leading to spike initiation in the proximal apical dendrite and then propagation of APs through the distal apical dendritic arbor (Fig. 8E). Presumably, the precisely-timed spikes generated by the proximal integration pathway regulate DD synaptic transmission by modulating NMDAR function (by temporally relieving $\mathrm{Mg}^{2+}$ blockade; Balu et al., 2007), creating a dual pathway system for facilitating DD inhibition known to rely preferentially on NMDARs (Isaacson and Strowbridge, 1998; Schoppa and Westbrook, 1999).

\section{Spike initiation zone in proximal apical dendrite}

Our results provide four lines of evidence that indicate AP initiation in GCs occurs in the proximal apical dendrite. First, we demonstrate that neither the distal apical arbor nor extensive basal dendrites are required to generate full-height APs in truncated GCs. Second, we find that, on average, truncated GCs with long apical dendrites (greater than the median length) had full-height APs, suggesting that voltage-gated $\mathrm{Na}^{+}$channels located within the first $80 \mu \mathrm{m}$ are sufficient to generate intact GC-like APs. We obtained a similar estimate of the initiation zone by measuring maximal rising phase slope: only truncated GCs with apical dendrites longer than the median length had intact GC-like membrane potential slopes. Third, we find the fastest AP-driven $\mathrm{Ca}^{2+}$ transients in the proximal apical dendrite of intact GCs (50-100 $\mu \mathrm{m}$ from the soma). And finally, we find the proximal apical dendrite (10-100 $\mu \mathrm{m}$ from the soma) is the only region in which focal TTX application reduced step-evoke discharges, also tested in intact GCs. The similarity in the estimates of the initiation zones from the comparative analysis of truncated GCs and pharmacological/photometry studies in intact GCs suggests that these results are not strongly biased by the absence of the distal apical arbor in truncated GCs.

The precision of our estimates of the AP initiation zone are limited by the number of different sites we can test with pharmacological probes and by the size of our pool of truncated GC recordings. We conclude that the AP initiation zone is within the proximal $100 \mu \mathrm{m}$ of the apical dendrite in GCs based on a conservative estimate from all four experimental approaches. Our methods cannot reveal whether $\mathrm{Na}^{+}$channels might be selectively enriched within hot spots within the proximal $100 \mu \mathrm{m}$ of the apical dendrite. However, we can eliminate the possibility that any potential hot spots are always in one fixed location as we occasionally find truncated GCs with relatively short apical dendrites (e.g., $50 \mu \mathrm{m}$ ) that have full-height APs and yet also find regions further out the apical dendrite $(50-100 \mu \mathrm{m}$ from the soma) where focal TTX application reduces step-evoked spike discharges. Finally, our results are limited to the question of where APs originate following somatic current injection, leaving for a future study the question of whether synaptic inputs initiate spikes in the same location.

Recent work has identified a requirement for $\mathrm{Na}_{\mathrm{V}} 1.2$ channels for AP generation in GCs (Nunes and Kuner, 2018), $\mathrm{Na}^{+}$channel subunits typically expressed at higher densities in axons in other cell types (Catterall et al., 2005; Lorincz and Nusser, 2010; Spratt et al., 2019). $\mathrm{Na}_{\mathrm{V}} 1.2$ subunits are expressed at higher levels in GC dendrites than cell bodies (Nunes and Kuner, 2018), consistent with our electrophysiological findings. However, $\mathrm{Na}_{\mathrm{V}} 1.2$ also appears to be expressed at high levels in distal segments of the GC apical dendrite, including in spine heads (Nunes and Kuner, 2018) This indicator, therefore, may reflect $\mathrm{Na}^{+}$channels involved both in spike initiation and active propagation of APs into the distal dendrites of GCs. Multiple imaging-based studies have focused on the functional role of voltage-gated $\mathrm{Na}^{+}$channels in distal GC dendrites and on dendritic spines (Zelles et al., 2006; Egger, 2008; Bywalez et al., 2015; Aghvami et al., 2019). Because of their different focus, these prior studies did not addressed the question of where APs initiate in GCs though Egger (2008) did report increased fractional changes in $\mathrm{Ca}^{2+}$ indicator responses as the sampling position along the apical dendrite was moved to more distal positions, consistent with our findings. Kosaka et al. (2008) identified $\mathrm{Na}^{+}$channel hot spots on proximal dendrites segments (within $50 \mu \mathrm{m}$ of the soma) of several OB interneuron subtypes, including GCs, based on immunohistochemical staining. These hot spot clusters appeared to colocalize with other markers proteins often enriched in axon initial segments, including $\beta$ IV spectrin and ankyrin G. The correspondence we find between T-type LVA $\mathrm{Ca}^{2+}$ currents and the site of presumed $\mathrm{Na}^{+}$spike initiation also parallels the organization of axon initial segments in other neuron types (Bender and Trussell, 2009; Bender et al., 2012). 
Low-threshold spikes promote shortlatency, precise firing in GCs

Our study finds three distinct functions of LVA $\mathrm{Ca}^{2+}$ currents in GCs. First, T-type $\mathrm{Ca}^{2+}$ currents located in the proximal apical dendrite appear to underlie short latency, low-threshold spikes that often trigger $\mathrm{Na}^{+}$spikes. Using this mechanism, GCs can overcome their tendency to spike late in response to depolarizing stimuli, a consequence of high-input resistance and long membrane time constants. Although many other neurons generate all-or-none spikes mediated by T-type $\mathrm{Ca}^{2+}$ currents (e.g., thalamic relay neurons; Jahnsen and Llinás, 1984; McCormick and Huguenard, 1992), this spike accelerating function of T-type $\mathrm{Ca}^{2+}$ currents may be more important in small interneurons, like GCs, than in larger relay neurons. In addition to decreasing spike latency, T-type $\mathrm{Ca}^{2+}$ currents in the proximal dendrite enhanced spike precision and amplified responses to trains of phasic depolarizations that mimicked rhythmic sensory-driven input.

Although other reports have examined LVA $\mathrm{Ca}^{2+}$ current in GC, we believe this report is the first to focus on the specific functional role of T-type $\mathrm{Ca}^{2+}$ current in the proximal apical dendrite. GCs also appear to express LVA Ca ${ }^{2+}$ current in their basal and distal apical dendrites (Egger et al., 2003, 2005; our unpublished observations). Spatial proximity to the AP initiation zone may explain why T-type $\mathrm{Ca}^{2+}$ current located in the proximal apical dendrite functions to accelerate spiking. In cartwheel cells (Bender and Trussell, 2009; Bender et al., 2012), pyramidal cells (Yu et al., 2010; Clarkson et al., 2017), and dentate GCs (Martinello et al., 2015) researchers have reported colocalization of T-type $\mathrm{Ca}^{2+}$ channels and high densities of voltage-gated $\mathrm{Na}^{+}$channels in axon initial segments; paralleling the colocalization we find in proximal dendrites in GCs. Based on these models, the enrichment of LVA $\mathrm{Ca}^{2+}$ currents near the spike generation zone of GCs could facilitate specific modulatory mechanisms that affect AP firing such as setting spike threshold.

\section{Functional significance}

There are at least two functional consequences to the location of AP initiation zone in the proximal apical dendrite. First, it suggests a novel route for synaptic processing exists in GCs (synaptic excitation of the basal dendrites, summation of proximal synaptic inputs in the cell body followed by spike initiation in the proxi-

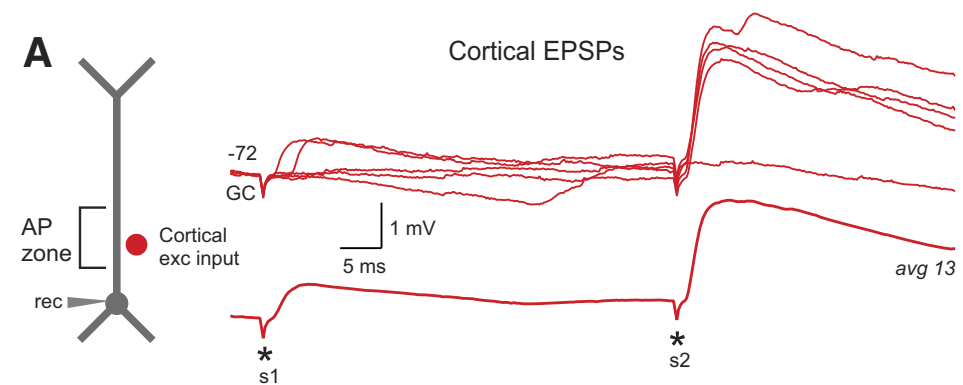

Dendrodendritic EPSPs
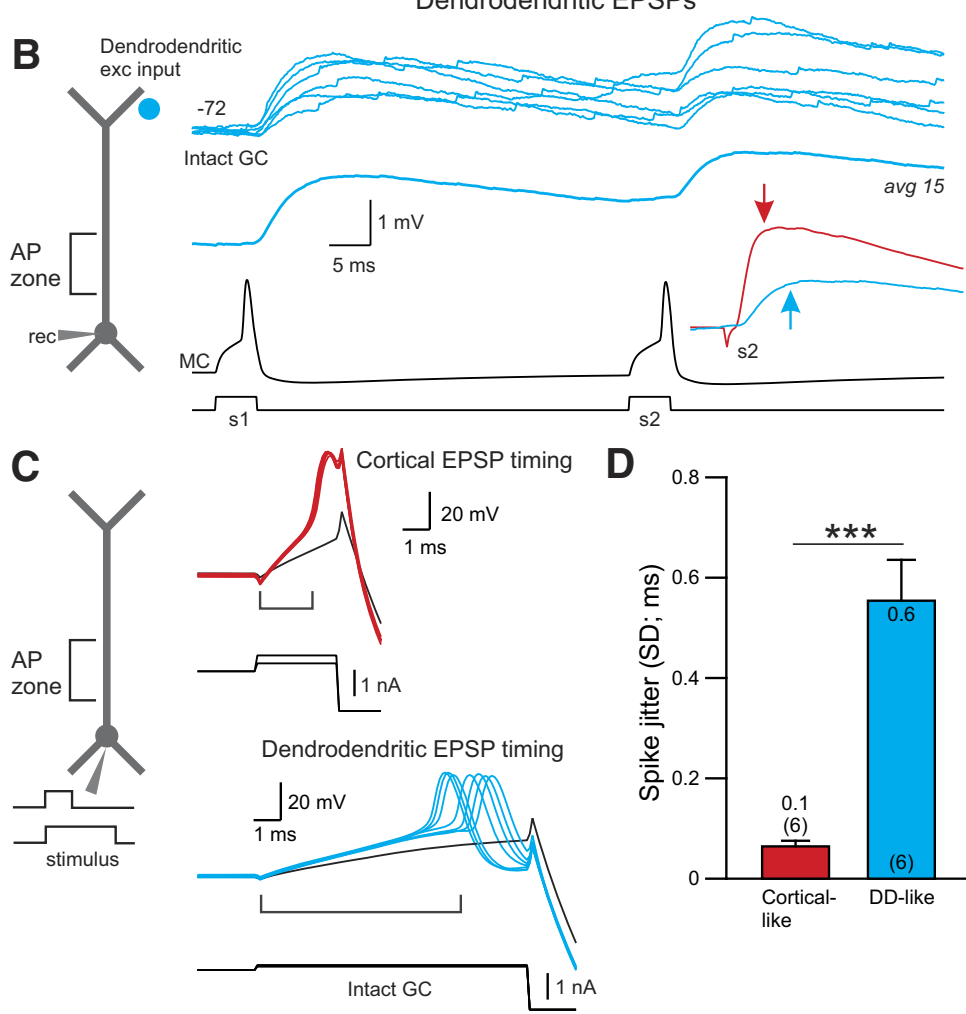

E
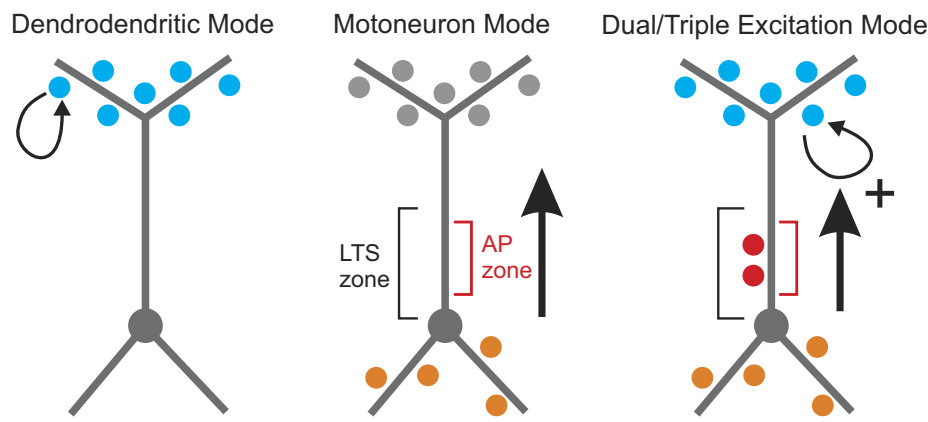

Figure 8. Higher spike precision in response to cortical-like EPSPs than dendrodendritic-like EPSPs. A, Example unitary (top) and average (bottom) responses to focal 2p-guided microstimulation of a presumptive cortical feedback excitatory input to an intact GC. Responses to two stimuli separated by $50 \mathrm{~ms}$ ( $20 \mathrm{~Hz}$; timing indicated by asterisks). Diagram at left indicates likely location of cortical feedback EPSP based on the position of the focal stimulating electrode (apical dendrite, $62 \mu \mathrm{m}$ from the soma). $\boldsymbol{B}$, Example dendrodendritic EPSPs recorded in an intact GC (blue traces). EPSPs evoked by activating APs in a presynaptic mitral cells within a dual simultaneous recording (black traces). Inset, Comparison of initial phase and peak (arrows) of average cortical (red) and dendrodendritic (blue) EPSPs using the same scaling. C, Example responses recorded in the same intact GC to short (3 ms; red) and long (10 ms; blue) duration current steps that mimic the rising phase kinetics of cortical and dendrodendritic EPSPs. Both steps adjusted to be just-suprathreshold; just-subthreshold responses indicated by black traces. D, Plot of AP jitter (latency SD) in just-suprathreshold responses to 3 and 10 ms duration current steps. ${ }^{* * *} p=9.047 E-4, T=6.026$, paired $t$ test. E, Summary diagram of three different activation modes for GCs: distal stimulation via dendrodendritic EPSPs (left), proximal stimulation via basal dendrite inputs (middle), and combined distal and proximal stimulation. Red circles indicate potential third excitatory pathway via cortical feedback inputs. Vertical brackets indicate likely dendritic zone required for maximal AP (red) and LTS (black) amplitude. Arrows indicate likely direction of AP propagation. 
mal apical dendrite) that parallels synaptic integration pathway in motoneurons and pyramidal cells. The primary difference in the $\mathrm{OB}$ is that the AP initiation zone appears to be located in a dendrite instead of a specialized region of the proximal axon. Although some evidence suggests that proximally-triggered APs can drive GABA release at distal DD synapses (Halabisky et al., 2000), it remains poorly understood how $\mathrm{Na}^{+}$spikes and locally-generated distal apical depolarization interact to generate lateral and self-inhibition of mitral and tufted cells. One appealing functional output of the proximal GC integration system may be generation of precisely-timed spikes that transiently relieve the tonic $\mathrm{Mg}^{2+}$ blockade of NMDARs governing DD transmission (Isaacson and Strowbridge, 1998; Schoppa and Westbrook, 1999; Balu et al., 2007). Our work also highlights the importance of the basal dendrite as an important site of synaptic excitation of GCs, though very little is known about the source and properties of these inputs. Also unknown is whether the OB contains GABAergic interneurons whose output connections target key elements of the proximal synaptic integration pathway (e.g., basket celllike interneurons that inhibit GC somata or axoaxonic-like interneurons that synapse near the spike initiation site on the proximal apical dendrite).

The second functional consequence of our results is highlighting the potentially important role the subclass of glutamatergic cortical feedback projections that synapse near the AP initiation zone. We show that simulated proximal excitatory inputs trigger precise spikes in GCs, unlike DD EPSPs that are heavily filtered before reaching the spike generation zone in the proximal apical dendrite. Excitatory inputs that target the proximal apical dendrite also bypass the somatic integration step that presumably regulates the ability of basalar inputs to trigger spikes. Although previous work demonstrated that one class of facilitating cortical inputs to the apical dendrite likely arises from anterior piriform cortex (Balu et al., 2007; Gao and Strowbridge, 2009; Boyd et al., 2012), further studies are required to define the specific termination zones and functional properties of the other major classes of glutamatergic feedback inputs to GCs, including projections from the anterior olfactory nucleus (Markopoulos et al., 2012) and posterior parietal cortex.

\section{References}

Abraham NM, Egger V, Shimshek DR, Renden R, Fukunaga I, Sprengel R, Seeburg PH, Klugmann M, Margrie TW, Schaefer AT, Kuner T (2010) Synaptic inhibition in the olfactory bulb accelerates odor discrimination in mice. Neuron 65:399-411.

Aghvami SS, Müller M, Araabi BN, Egger V (2019) Coincidence detection within the excitable rat olfactory bulb granule cell spines. J Neurosci 39:584-595.

Balu R, Pressler RT, Strowbridge BW (2007) Multiple modes of synaptic excitation of olfactory bulb granule cells. J Neurosci 27:5621-5632.

Bender KJ, Trussell LO (2009) Axon initial segment $\mathrm{Ca}^{2+}$ channels influence action potential generation and timing. Neuron 61:259-271.

Bender KJ, Uebele VN, Renger JJ, Trussell LO (2012) Control of firing patterns through modulation of axon initial segment T-type calcium channels. J Physiol 590:109-118.

Boyd AM, Sturgill JF, Poo C, Isaacson JS (2012) Cortical feedback control of olfactory bulb circuits. Neuron 76:1161-1174.

Burton SD, Urban NN (2015) Rapid feedforward inhibition and asynchronous excitation regulate granule cell activity in the mammalian main olfactory bulb. J Neurosci 35:14103-14122.

Bywalez WG, Patirniche D, Rupprecht V, Stemmler M, Herz AV, Pálfi D, Rózsa B, Egger V (2015) Local postsynaptic voltage-gated sodium channel activation in dendritic spines of olfactory bulb granule cells. Neuron 85:590-601.

Cang J, Isaacson JS (2003) In vivo whole-cell recording of odor-evoked synaptic transmission in the rat olfactory bulb. J Neurosci 23:4108-4116.
Carleton A, Petreanu LT, Lansford R, Alvarez-Buylla A, Lledo PM (2003) Becoming a new neuron in the adult olfactory bulb. Nat Neurosci 6:507-518.

Castillo PE, Carleton A, Vincent JD, Lledo PM (1999) Multiple and opposing roles of cholinergic transmission in the main olfactory bulb. J Neurosci 19:9180-9191.

Catterall WA, Goldin AL, Waxman SG (2005) International union of pharmacology: XLVII. Nomenclature and structure-function relationships of voltage-gated sodium channels. Pharmacol Rev 57:397-409.

Cembrowski MS, Logan SM, Tian M, Jia L, Li W, Kath WL, Riecke H, Singer JH (2012) The mechanisms of repetitive spike generation in an axonless retinal interneuron. Cell Rep 1:155-166.

Clarkson RL, Liptak AT, Gee SM, Sohal VS, Bender KJ (2017) D3 receptors regulate excitability in a unique class of prefrontal pyramidal cells. J Neurosci 37:5846-5860.

Cleland TA (2014) Construction of odor representations by olfactory bulb microcircuits. Prog Brain Res 208:177-203.

Cook PB, Werblin FS (1994) Spike initiation and propagation in wide field transient amacrine cells of the salamander retina. J Neurosci 14:3852-3861.

de Olmos J, Hardy H, Heimer L (1978) The afferent connections of the main and the accessory olfactory bulb formations in the rat: an experimental HRP-study. J Comp Neurol 181:213-244.

DiGregorio DA, Peskoff A, Vergara JL (1999) Measurement of action potential-induced presynaptic calcium domains at a cultured neuromuscular junction. J Neurosci 19:7846-7859.

Egger V (2008) Synaptic sodium spikes trigger long-lasting depolarizations and slow calcium entry in rat olfactory bulb granule cells. Eur J Neurosci 27:2066-2075.

Egger V, Svoboda K, Mainen ZF (2003) Mechanisms of lateral inhibition in the olfactory bulb: efficiency and modulation of spike-evoked calcium influx into granule cells. J Neurosci 23:7551-7558.

Egger V, Svoboda K, Mainen ZF (2005) Dendrodendritic synaptic signals in olfactory bulb granule cells: local spine boost and global low-threshold spike. J Neurosci 25:3521-3530.

Escobar AL, Monck JR, Fernandez JM, Vergara JL (1994) Localization of the site of $\mathrm{Ca}^{2+}$ release at the level of a single sarcomere in skeletal muscle fibres. Nature 367:739-741.

Eyre MD, Kerti K, Nusser Z (2009) Molecular diversity of deep short-axon cells of the rat main olfactory bulb. Eur J Neurosci 29:1397-1407.

Friedman D, Strowbridge BW (2003) Both electrical and chemical synapses mediate fast network oscillations in the olfactory bulb. J Neurophysiol 89:2601-2610.

Gao Y, Strowbridge BW (2009) Long-term plasticity of excitatory inputs to granule cells in the rat olfactory bulb. Nat Neurosci 12:731-733.

Haberly LB, Price JL (1978) Association and commissural fiber systems of the olfactory cortex of the rat. J Comp Neurol 178:711-740.

Halabisky B, Friedman D, Radojicic M, Strowbridge BW (2000) Calcium influx through NMDA receptors directly evokes GABA release in olfactory bulb granule cells. J Neurosci 20:5124-5134.

Hyde RA, Strowbridge BW (2012) Mnemonic representations of transient stimuli and temporal sequences in the rodent hippocampus in vitro. Nat Neurosci 15:1430-1438.

Inoue T, Strowbridge BW (2008) Transient activity induces a long-lasting increase in the excitability of olfactory bulb interneurons. J Neurophysiol 99:187-199.

Isaacson JS, Strowbridge BW (1998) Olfactory reciprocal synapses: dendritic signaling in the CNS. Neuron 20:749-761.

Jahnsen H, Llinás R (1984) Ionic basis for the electro-responsiveness and oscillatory properties of guinea-pig thalamic neurones in vitro. J Physiol 349:227-247.

Kavalali ET, Zhuo M, Bito H, Tsien RW (1997) Dendritic $\mathrm{Ca}^{2+}$ channels characterized by recordings from isolated hippocampal dendritic segments. Neuron 18:651-663.

Kelsch W, Lin CW, Lois C (2008) Sequential development of synapses in dendritic domains during adult neurogenesis. Proc Natl Acad Sci U S A 105:16803-16808.

Kosaka T, Komada M, Kosaka K (2008) Sodium channel cluster, $\beta$ IVspectrin and ankyrinG positive "hot spots" on dendritic segments of parvalbumin-containing neurons and some other neurons in the mouse and rat main olfactory bulbs. Neurosci Res 62:176-186.

Kullmann PH, Kandler K (2008) Dendritic $\mathrm{Ca}^{2+}$ responses in neonatal lat- 
eral superior olive neurons elicited by glycinergic/GABAergic synapses and action potentials. Neuroscience 154:338-345.

Larimer P, Strowbridge BW (2008) Nonrandom local circuits in the dentate gyrus. J Neurosci 28:12212-12223.

Lee JH, Gomora JC, Cribbs LL, Perez-Reyes E (1999) Nickel block of three cloned T-type calcium channels: low concentrations selectively block alpha1H. Biophys J 77:3034-3042.

Lorincz A, Nusser Z (2010) Molecular identity of dendritic voltage-gated sodium channels. Science 328:906-909.

Losonczy A, Makara JK, Magee JC (2008) Compartmentalized dendritic plasticity and input feature storage in neurons. Nature 452:436-441.

Magee JC, Johnston D (1995) Characterization of single voltage-gated $\mathrm{Na}^{+}$ and $\mathrm{Ca}^{2+}$ channels in apical dendrites of rat CA1 pyramidal neurons. J Physiol 487:67-90.

Markopoulos F, Rokni D, Gire DH, Murthy VN (2012) Functional properties of cortical feedback projections to the olfactory bulb. Neuron 76:1175-1188.

Martinello K, Huang Z, Lujan R, Tran B, Watanabe M, Cooper EC, Brown DA, Shah MM (2015) Cholinergic afferent stimulation induces axonal function plasticity in adult hippocampal granule cells. Neuron 85:346-363.

Matsutani S, Yamamoto N (2004) Postnatal development of dendritic spines on olfactory bulb granule cells in rats. J Comp Neurol 473:553-561.

McCormick DA, Huguenard JR (1992) A model of the electrophysiological properties of thalamocortical relay neurons. J Neurophysiol 68:1384-1400.

Nakamura Y, Harada H, Kamasawa N, Matsui K, Rothman JS, Shigemoto R, Silver RA, DiGregorio DA, Takahashi T (2015) Nanoscale distribution of presynaptic $\mathrm{Ca}^{2+}$ channels and its impact on vesicular release during development. Neuron 85:145-158.

Nunes D, Kuner T (2018) Axonal sodium channel NaV1.2 drives granule cell dendritic GABA release and rapid odor discrimination. PLoS Biol 16:e2003816.

Obejero-Paz CA, Gray IP, Jones SW (2008) $\mathrm{Ni}^{2+}$ block of CaV3.1 $(\alpha 1 \mathrm{G})$ T-type calcium channels. J Gen Physiol 132:239-250.

Petreanu L, Alvarez-Buylla A (2002) Maturation and death of adult-born olfactory bulb granule neurons: role of olfaction. J Neurosci 22:6106-6113.

Pinato G, Midtgaard J (2003) Regulation of granule cell excitability by a low-threshold calcium spike in turtle olfactory bulb. J Neurophysiol 90: 3341-3351.

Pinato G, Midtgaard J (2005) Dendritic sodium spikelets and low-threshold calcium spikes in turtle olfactory bulb granule cells. J Neurophysiol 93: $1285-1294$.

Pressler RT, Strowbridge BW (2006) Blanes cells mediate persistent feedforward inhibition onto granule cells in the olfactory bulb. Neuron 49:889-904.

Pressler RT, Strowbridge BW (2017) Direct recording of dendrodendritic excitation in the olfactory bulb: divergent properties of local and external glutamatergic inputs govern synaptic integration in granule cells. J Neurosci 37:11774-11788.

Pressler RT, Rozman PA, Strowbridge BW (2013) Voltage-dependent intrinsic bursting in olfactory bulb Golgi cells. Learn Mem 20:459-466.

Price JL, Powell TP (1970a) The morphology of the granule cells of the olfactory bulb. J Cell Sci 7:91-123.
Price JL, Powell TP (1970b) The synaptology of the granule cells of the olfactory bulb. J Cell Sci 7:125-155.

Rall W, Shepherd GM, Reese TS, Brightman MW (1966) Dendrodendritic synaptic pathway for inhibition in the olfactory bulb. Exp Neurol 14:44-56.

Sanger F, Nicklen S, Coulson AR (1977) DNA sequencing with chainterminating inhibitors. Proc Natl Acad Sci U S A 74:5463-5467.

Schmidt LJ, Strowbridge BW (2014) Modulation of olfactory bulb network activity by serotonin: synchronous inhibition of mitral cells mediated by spatially localized GABAergic microcircuits. Learn Mem 21:406-416.

Schneider SP, Macrides F (1978) Laminar distributions of internuerons in the main olfactory bulb of the adult hamster. Brain Res Bull 3:73-82.

Schoppa NE, Westbrook GL (1999) Regulation of synaptic timing in the olfactory bulb by an A-type potassium current. Nat Neurosci 2:1106-1113.

Shepherd GM, Chen WR, Willhite D, Migliore M, Greer CA (2007) The olfactory granule cell: from classical enigma to central role in olfactory processing. Brain Res Rev 55:373-382.

Shipley MT, Adamek GD (1984) The connections of the mouse olfactory bulb: a study using orthograde and retrograde transport of wheat germ agglutinin conjugated to horseradish peroxidase. Brain Res Bull 12:669-688.

Spratt PWE, Ben-Shalom R, Keeshen CM, Burke KJ Jr, Clarkson RL, Sanders SJ, Bender KJ (2019) The autism-associated gene SCN2a contributes to dendritic excitability and synaptic function in the prefrontal cortex. Neuron 103:673-685.e5.

Stuart G, Schiller J, Sakmann B (1997a) Action potential initiation and propagation in rat neocortical pyramidal neurons. J Physiol 505:617-632.

Stuart G, Spruston N, Sakmann B, Häusser M (1997b) Action potential initiation and backpropagation in neurons of the mammalian CNS. Trends Neurosci 20:125-131.

Sun Q, Srinivas KV, Sotayo A, Siegelbaum SA (2014) Dendritic $\mathrm{Na}^{+}$spikes enable cortical input to drive action potential output from hippocampal CA2 pyramidal neurons. eLife 3:e04551.

Whitman MC, Greer CA (2007) Synaptic integration of adult-generated olfactory bulb granule cells: basal axodendritic centrifugal input precedes apical dendrodendritic local circuits. J Neurosci 27:9951-9961.

Wu C, Ivanova E, Cui J, Lu Q, Pan ZH (2011) Action potential generation at an axon initial segment-like process in the axonless retinal AII amacrine cell. J Neurosci 31:14654-14659.

Youngstrom IA, Strowbridge BW (2015) Respiratory modulation of spontaneous subthreshold synaptic activity in olfactory bulb granule cells recorded in awake, head-fixed mice. J Neurosci 35:8758-8767.

Yu Y, Maureira C, Liu X, McCormick D (2010) P/Q and N channels control baseline and spike-triggered calcium levels in neocortical axons and synaptic boutons. J Neurosci 30:11858-11869.

Yu Y, Migliore M, Hines ML, Shepherd GM (2014) Sparse coding and lateral inhibition arising from balanced and unbalanced dendrodendritic excitation and inhibition. J Neurosci 34:13701-13713.

Zelles T, Boyd JD, Hardy AB, Delaney KR (2006) Branch-specific $\mathrm{Ca}^{2+}$ influx from $\mathrm{Na}^{+}$-dependent dendritic spikes in olfactory granule cells. J Neurosci 26:30-40. 